\title{
X-ray and multiwavelength view of NGC 4278
}

\section{A LINER-Seyfert connection?}

\author{
G. Younes ${ }^{1}$, D. Porquet ${ }^{1}$, B. Sabra ${ }^{2}$, N. Grosso ${ }^{1}$, J. N. Reeves ${ }^{3}$, and M. G. Allen ${ }^{1}$ \\ ${ }^{1}$ Observatoire astronomique de Strasbourg, Université de Strasbourg, CNRS, UMR 7550, 11 rue de l'Université, \\ 67000 Strasbourg, France \\ e-mail: younes@astro.u-strasbg.fr \\ 2 Department of Sciences, Notre Dame University-Louaize, PO Box 72, Zouk Mikael, Lebanon \\ 3 Astrophysics Group, School of Physical \& Geographical Sciences, Keele University, Keele, Staffordshire ST5 5BG, UK
}

Received 7 March 2010 / Accepted 19 April 2010

\begin{abstract}
Context. The emission mechanism responsible for the bulk of energy from radio to X-rays in low ionization emission line regions (LINERs) and low luminosity active galactic nuclei (LLAGN) has been long debated. Based on UV to X-ray and radio to UV flux ratios, some argue that LINERs/LLAGN are a scaled-down version of their more luminous predecessors Seyfert galaxies. Others, based on the lack of X-ray short (hours) time-scale variability, the non detection of an iron line at $6.4 \mathrm{keV}$, and the faint UV emission compared to typical AGNs, suggest the truncation of the classical thin accretion disk in the inner regions of the AGN where a radiatively inefficient accretion flow (RIAF) structure forms.

Aims. We investigate the LINER-Seyfert connection by studying the unabsorbed LINER galaxy NGC 4278 that accretes at a low rate $\left(L_{\text {bol/Edd }} \sim 7 \times 10^{-6}\right.$ ) but exhibits a broad $\mathrm{H} \alpha$ line, and a point-like nucleus in radio, optical, UV and X-rays.

Methods. We analyzed one XMM-Newton and seven Chandra X-ray observations of NGC 4278 spread over a three year period, allowing the study of the X-ray variability at different time-scales (hours, months, years). We also examined the radio to X-ray spectral energy distribution to constrain the accretion mode in the nucleus of NGC 4278.

Results. Long time-scale (months) variability is observed where the flux increased by a factor of $\sim 3$ on a time-scale of a few months and by a factor of 5 between the faintest and the brightest observation separated by $\sim 3$ years. During the XMM-Newton observation, where the highest flux level is detected, we found a $10 \%$ flux increase on a short time-scale of a few hours, while the light curves for the different Chandra observations do not show short time-scale (minutes to hours) variability. A combination of an absorbed power law $\left(N_{\mathrm{H}} \approx 10^{20} \mathrm{~cm}^{-2}, \Gamma=2.2_{-0.2}^{+0.1}\right)$ plus a thermal component $(k T \approx 0.6 \mathrm{keV})$ were able to fit the Chandra spectra. The $X M M-N e w t o n$ spectra, where the highest X-ray flux is detected, are well fitted with an absorbed power-law with no need for a thermal component as the emission from the power-law component is dominant. The power-law photon index is $\sim 2.1$ and the hydrogen column density is of the order of $10^{20} \mathrm{~cm}^{-2}$. Neither a narrow nor a broad $\mathrm{Fe} \mathrm{K} \alpha$ emission line at $6.4 \mathrm{keV}$ are detected with a $22 \mathrm{eV}$ and $118 \mathrm{eV}$ upper limits derived on their equivalent widths. We derive optical fluxes from archival HST ACS observations and detected optical variability on time-scales of years. For the first time for this source, thanks to the optical/UV monitor on board XMM-Newton, we obtained simultaneous UV and X-ray flux measurements. We constructed SEDs based on simultaneous or quasi simultaneous observations and compared them to LINER, radio-loud, and radio-quiet quasar SEDs. We find that at a low X-ray flux the NGC 4278 SED resembles that of typical LINER sources where the radio to X-ray emission can be considered as originating from a jet and/or RIAF, whereas at a high X-ray flux, NGC 4278 SED is more like a low luminosity Seyfert SED. Consequently, NGC 4278 could exhibit both LINER and Seyfert nuclear activity depending on the strength of its X-ray emission.
\end{abstract}

Key words. accretion, accretion disks - Galaxy: nucleus - galaxies: active - galaxies: individual: NGC 4278

\section{Introduction}

Low-ionization nuclear emission line regions (LINERs) were first identified by Heckman (1980) as a class of galaxies with optical spectra dominated by emission lines from low ionization species. The ionization mechanism is yet poorly known and could be explained, either in terms of starbursts (Alonso-Herrero et al.2000) or, more consistently, being due to a low luminosity active galactic nuclei (LLAGN) (Terashima et al. 2000; Ho et al. 1993). This latter idea rises from the detection of broad $\mathrm{H} \alpha \mathrm{com}$ ponents in an important fraction of LINER sources (noted as LINER 1.9 (hereinafter LINERI) objects, Ho et al. 1997), and/or a point-like UV or X-ray source at the nucleus. If the luminosity scales with the accretion rate, the study of the LINER nucleus define a supreme pattern for probing low accretion rate physics around supermassive black holes (SMBHs).

How similar are these LINERI sources to classical luminous Seyfert and quasar galaxies? Based on observational properties, the weakness or absence of a big blue bump feature at UV wavelengths ( $\mathrm{Ho}$ 2008) usually detected in AGN (Malkan \& Sargent 1982; Sanders et al. 1989; Koratkar \& Blaes 1999), the lack of a broad $\mathrm{Fe} \mathrm{K} \alpha$ emission line at $6.4 \mathrm{keV}$ (Terashima et al. 2002; Ptak et al. (2004), except for the peculiar LINER NGC 1052 (Brenneman et al. 2009), and last but not least, the 
lack of short time-scale (minutes to hours) X-ray variability have been attributed to an intrinsic difference in LINERsI/LLAGN central engine as opposed to normal Seyfert galaxies. One proposed scenario is that accretion in LINERsI/LLAGN is radiatively inefficient, advection dominated compared to the typical geometrically thin optically thick accretion disks present in luminous AGN (Narayan 2005).

However, more recently $\mathrm{Maoz}$ et al. (2005) revealed UV variability in the nuclei of a sample of 17 LINERs observed with the HST. By combining these results with non-simultaneous radio and X-ray observations, Maoz (2007) demonstrated that the UV/X-ray luminosity ratios are similar to those of Seyfert 1 nuclei and pointed out that LLAGN may be a scaled-down version of Seyfert galaxies where a thin accretion disk exists. This idea is supported by Pian et al. (2010) who studied simultaneous UV to X-ray observations of four LINER nuclei observed with the XRT and the UVOT on-board the Swift telescope. They discovered short-time scale (half a day) X-ray variability in two of their objects and showed that the UV to X-ray flux ratios are consistent with those of more luminous AGN.

The elliptical galaxy NGC 4278 (distance of $16.7 \mathrm{Mpc}$; Tonry et al. 2001, scaled to $H_{0}=70 \mathrm{~km} \mathrm{~s}^{-1} \mathrm{Mpc}^{-1}$ ) has been studied extensively at different wavelengths. At radio wavelength, a compact non-thermal radio source has been detected at 6 and $18 \mathrm{~cm}$ (Jones et al. 1984). Nagar et al. (2005) reported two-sided radio emission on subparsec scales in the form of twin jets emerging from a central compact component $\left(T_{B}=1.5 \times\right.$ $\left.10^{9} \mathrm{~K}\right)$. Ho et al. (1997) classified NGC 4278 as a type 1.9 LINER from the definite detection of a broad $\mathrm{H} \alpha$ line, with

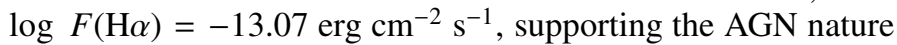
of the nuclear engine. Moreover, an unresolved compact nuclear source has been detected in HST WFPC2 images (Capetti et al. 2000). Finally, Ho et al. (2001), after studying a Chandra $1.4 \mathrm{ks}$ snapshot taken in April 2000, gave NGC 4278 a class (I) X-ray morphology, showing a dominant nuclear source. Terashima \& Wilson (2003) fit the $0.5-8 \mathrm{keV}$ spectrum obtained during this same Chandra snapshot with a power law modified by absorption and found a $2-10 \mathrm{keV}$ corrected luminosity of $9.1 \times$ $10^{39} \mathrm{erg} \mathrm{s}^{-1}$. González-Martín et al. (2009) find a $0.5-10 \mathrm{keV}$ corrected luminosity of $5.6 \times 10^{39} \mathrm{erg} \mathrm{s}^{-1}$ after studying a $\sim 100$ ks Chandra observation taken in March 2006.

The mass of a black hole in the nucleus of NGC 4278, derived from the $M-\sigma$ relation (Tremaine et al. 2002), is $3.09 \pm$ $0.54 \times 10^{8} M_{\odot}$ Wang \& Zhang 2003; Chiaberge et al. 2005). This leads to a $L_{\mathrm{Edd}} \approx 3.9 \times 10^{46} \mathrm{erg} \mathrm{s}^{-1}$; assuming that $L_{\mathrm{Edd}}=1.25 \times 10^{38}\left(M_{\mathrm{BH}} / M_{\odot}\right) \mathrm{erg} \mathrm{s}^{-1}$. This implies a very low $L_{\mathrm{X}} / L_{\mathrm{Edd}}$ ratio of $2 \times 10^{-7}$.

In this paper we report a timing and X-ray spectral study of the LINER galaxy NGC 4278 observed with XMM-Newton and Chandra. We describe in Sect. 2 the X-ray observations and the data reduction. Timing and spectral $\mathrm{X}$-ray results as well as optical (HST/ACS) and UV (XMM-Newton/OM) results are presented in Sect. 3. In Sect. 4, we describe the construction of the NGC 4278 spectral energy distribution. We discuss in Sect. 5 our results in the context of the LINER-Seyfert connection. The main results are summarized in Sect. 6.

\section{X-ray observations and data reduction}

\subsection{Chandra observations}

NGC 4278 was observed seven times with the Chandra observatory, with a first $1.4 \mathrm{ks}$ snapshot taken in 2000 April 20 (Ho et al. 2001). The following six observations were taken over a two year interval starting on 2005 February 03 and ended on 2007 April 20. The seven observations were obtained with the spectroscopic array (ACIS-S; Weisskopf et al. 2002) where the nucleus was placed on the aim point of the ACIS-S3 backilluminated chip. They were taken in Very Faint mode to increase their sensitivity. All of the observations are Chandra archival data obtained from chaser]. The log of the Chandra observations are listed in Table 1.

All seven observations were reduced and analyzed in a systematic, homogeneous way using the CIAO software package version 4.2, Chandra calibration database, CALDB, version 4.2, and the ACIS extract (AE) software package version 3.175: (Broos et al. 2010). We started by using the level 1 event file produced by the Chandra X-ray center (CXC) to suppress the position randomization applied by the CXC standard data processing when creating a level 2 event file. We also corrected for the effects of charge-transfer inefficiency on event energies and grades. We filtered for bad event grades (only ASCA grades 0, 2, 3,4 and 6 are accepted) and hot columns to take account of several potential issues such as cosmic rays and bad pixels. Good time intervals, supplied by the pipeline, were applied to the final product 3 .

In order to get the X-ray position for the sources in the ACIS-S field of view, we used a wavelet transform detection algorithm, the wavdetect program within the CIAO data analysis system (Freeman et al.2002). The output file of the previous procedure is input to the AE software. The AE package is used to refine source positions, extract source photons, construct local backgrounds, extract source, and background spectra (see below), compute redistribution matrix files (RMFs) and auxiliary response files (ARFs), by spawning the mkarf and mkacisrmf routines of CIAO, and perform spectral grouping and fitting. We focus here on the X-ray emission from the nuclear source (for information on the X-ray source population of NGC 4278 see Brassington et al. 2009).

Source events are extracted around the source centroid, inside a polygonal shape of the local PSF, generated by ChaRT4 at the energy $1.497 \mathrm{keV}$ using the ae_make_psf tool implemented in AE. Background region was defined on the brightest of the seven observations (obs ID: 4741) following the AE procedure. This background region is an annular region centered on the source position where the inner radius delimit $1.1 \times 99 \%$ encircled energy radius and the outer radius is set such that the background includes 200 counts. This number is obtained from a special image where all events within the $\sim 1.1 \times 99 \%$ PSF circles of all the sources in the field were excluded (swiss cheese image). This background region is then generated to the other observations in order to have a consistent and coherent measurement of the diffuse/unresolved X-ray emission between the seven observations for a homogeneous spectral analysis.

We find that all of the seven Chandra observations suffer from pile-up. X-ray data affected by pile-up can mimic spectral and timing variability. Therefore, pile-up was accounted for by excluding the core of the PSF (see Appendix A for more details). We decided not to use the first $1.4 \mathrm{ks}$ Chandra snapshot observation of NGC 4278. Indeed, after removing the piled-core, not enough counts remained to build a useful spectrum. We use

\footnotetext{
1 http://cda.harvard.edu/chaser/Descriptions

2 The ACIS Extract software package and User's Guide are available at http://www.astro.psu.edu/xray/acis/acis_analysis.html

${ }^{3}$ Description on how to create a new level 2 event file can be found at http://cxc.harvard.edu/ciao/threads/createL2/

${ }^{4}$ http://cxc.harvard.edu/chart/
} 
Table 1. Log of the X-ray observations.

\begin{tabular}{ccccc}
\hline \hline Satellite & Instrument & Start date & Obs. ID & Exposure time (ks) \\
\hline Chandra $^{2}$ & ACIS-S & 2000 April 20 & 398 & 1.4 \\
XMM-Newton & EPIC & 2004 May 23 & 205010101 & $34 / 35.5 / 35.5$ \\
Chandra $_{\text {Chandra }}$ & ACIS-S & 2005 February 02 & 4741 & 37 \\
Chandra & ACIS-S & 2006 March 16 & 7077 & 110 \\
Chandra & ACIS-S & 2006 July 25 & 7078 & 51 \\
Chandra & ACIS-S & 2006 October 24 & 7079 & 105 \\
Chandra & ACIS-S & 2007 February 20 & 7081 & 110 \\
\hline
\end{tabular}

Notes. ${ }^{(a)}$ Exposure time for pn/MOS1/MOS2 respectively.

the tool dmextract, called by the AE software, to create a source spectrum over the energy range $0.5-8 \mathrm{keV}$. We used the tool ae_group_spectrum implemented in $\mathrm{AE}$ to group the spectra. Channels between 0.5 and $8 \mathrm{keV}$ are grouped to have a three sigma $(3 \sigma)$ signal to noise ratio, which corresponds to a minimum of 20 counts per bin, to enable the use of $\chi^{2}$ statistics in the spectral analysis.

\subsection{XMM-Newton observation}

The log of the XMM-Newton observation is listed in Table 1 NGC 4278 was observed by XMM-Newton on 2004 May 23. The EPIC-pn (Strüder et al. 2001) and MOS (Turner et al. 2001) cameras were operated in Imaging, Prime Full Frame mode using the thin filter and medium filter respectively (exposure time about 34 and $35.5 \mathrm{ks}$, respectively). The Reflection Grating Spectrometers show few counts therefore they were not included in our analysis. The optical/UV monitor (OM) instrument (Mason et al. 2001) was operated in imaging mode with the $U V W 1$ and the UVM2 filters (effective $\lambda$ of 2910 and $2310 \AA$, respectively). All data products were obtained from the XMM-Newton science archive (XSA) 5 and reduced using the Science Analysis System (SAS) version 9.0. The EPIC data were not affected by pile-up, and no severe intervals of enhanced solar activity were present. Data are selected using event patterns 0-4 and 0-12 for pn and MOS, respectively, during only good X-ray events ("FLAG = 0").

We extract source events from a circle of $10^{\prime \prime}$ radius centered on the source. Background events are extracted from a sourcefree circle of the same radius on the same CCD as the source. We generated response matrix files using the SAS task $r m f$ gen, while ancillary response files were generated using the SAS task arfgen. The EPIC spectra were created in the energy range $0.5-8 \mathrm{keV}$ to enable flux and model-parameter comparison with Chandra spectra6. They were grouped using the FTOOLS grppha tool to have a minimum of 20 counts per bin to allow the use of the $\chi^{2}$ statistic. The SAS task omichain was used to process the OM data.

5 http://xmm.esac.esa.int/xsa/index.shtml

6 In fact, the contribution from the surrounding medium around NGC 4278 that we calculated using the Chandra images is unknown below $0.5 \mathrm{keV}$ as we extracted the spectra in the $0.5-8 \mathrm{keV}$ range, see Sect. 3.3.2 for more details.

\section{Results of the nucleus study}

\subsection{Lightcurves and hardness ratios}

Light curves for the six Chandra observations were extracted in the energy range $0.5-10 \mathrm{keV}$ from the pile-up free wings of the PSF. To enable the comparison between the different observations, the net count rate of each is then corrected for the excluded fraction of the PSF. The hardness ratio is defined as, $H R=(H-S) /(H+S)$, where $S$ is the count rate in the soft $0.5-2 \mathrm{keV}$ band and $H$ is the count rate in the hard $2-10 \mathrm{keV}$ band. Figure 1 shows the $0.5-10 \mathrm{keV}$ light curves and the hardness-ratios of the different observations. No clear-cut short timescale, minutes to hours, variability is detected in any of the observations. We conducted a Kolmogorov-Smirnov, K-S, test to check more accurately any variability in the source count rate within each observation. We find a K-S test probability $>17 \%$ in the six observations that the nuclear emission originates from a constant source. Therefore, NGC 4278 is considered as constant on short (minutes to hours) timescale. On the other hand, long timescale (months) variability is observed between the different observations. The hardness ratio in all of the six observations indicate that the source is in a soft state being constant during each observation and between the observations as well implying similar spectral characteristics.

We extracted an EPIC-pn light curve from a $10^{\prime \prime}$ circle centered on the source. We used the tool epiclccorl to correct for good time intervals (GTIs), dead time, and for background subtraction. On account of the low angular resolution of $X M M-N e w t o n$ compared to Chandra, events from the $10^{\prime \prime}$ circle are contaminated by emission from point-like sources and diffuse emission (Fig. 2). Nevertheless, we show in Appendix B that emission from the central source is dominant and all the surrounding sources only contribute in $2 \%$ of the total X-ray flux. Figure 3 shows the pn light curve and the associated hardness ratio. According to a K-S test, NGC 4278 shows variability during the XMM-Newton observation with a $\mathrm{K}-\mathrm{S}$ probability of $0.8 \%$ that the emission comes from a constant source. This variability is clear at the beginning of the observation where we detect a $10 \%$ increase over a period of $\sim 1 \mathrm{~h}$.

Finally, we calculated the normalized "excess variance", $\sigma_{\text {rms }}^{2}$ defined as in Nandra et al. (1997) and Turner et al. (1999) for the seven light curves, in order to check more accurately

\footnotetext{
${ }^{7}$ http://xmm.esa.int/sas/current/doc/epiclccorr/ epiclccorr.html
} 

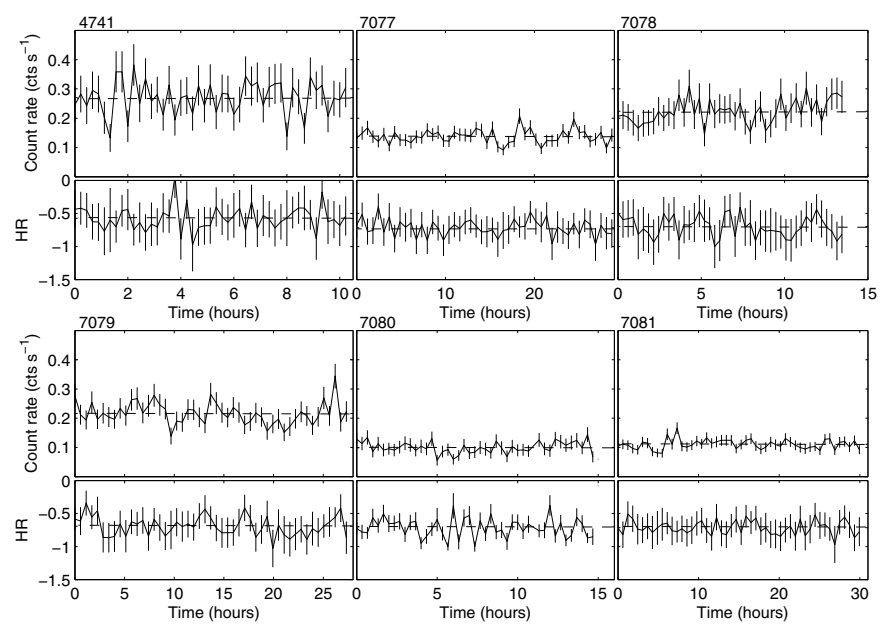

Fig. 1. Light curves of the six Chandra observations along with their hardness ratios defined as $H R=(H-S) /(H+S)$, where $S$ is the count rate in the soft $0.5-2 \mathrm{keV}$ band and $H$ is the count rate in the hard 2-10 keV band. Light curves were binned to have 50 groups with a signal to noise ratio greater than 10 . The dashed lines show the averages of the count rates and hardness ratios.
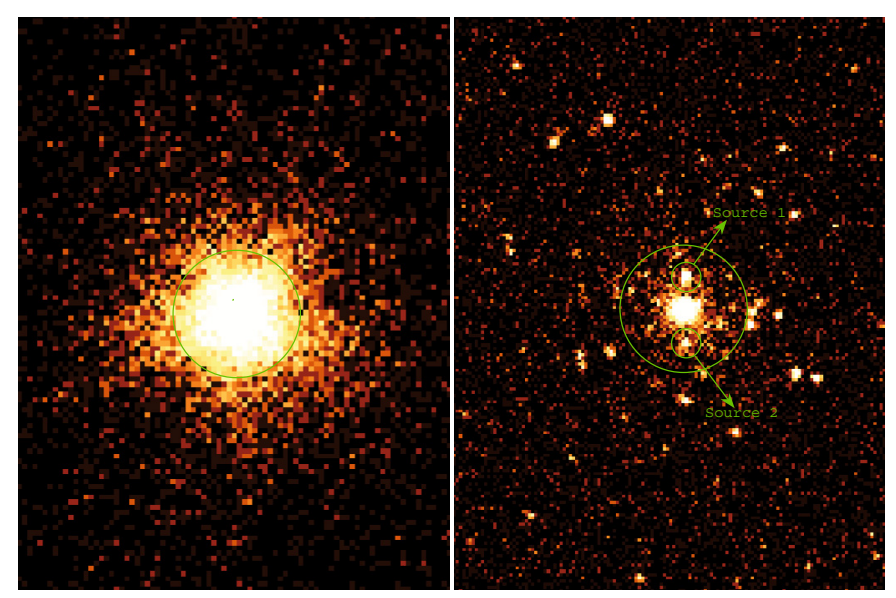

Fig. 2. Left panel: the XMM-Newton/MOS1 image of NGC 4278 with a $10^{\prime \prime}$ circle centered on the nucleus. Right panel: the Chandra image (obs ID: 7077) with a $10^{\prime \prime}$-radius circle centered on the nucleus. The lower angular resolution of XMM-Newton has blended the central source, X-ray point-like sources, and diffuse emission, detected with Chandra, in one point-like source.

any possible variability during each observation. We find that $\sigma_{\text {rms }}^{2}<0.01$ in all of the observations with an error $<15 \%$ and $\sim 20 \%$ for the Chandra and XMM-Newton observations respectively. This result is discussed in Sect. 5.2.

\subsection{Spectral analysis}

The spectral analysis was performed using XSPEC Arnaud 1996) version 12.5.1. The updated photo-electric cross sections and the revised solar abundances of Wilms et al. (2000) are used throughout to account for absorption by neutral gas. An absorbed galactic column density derived from Kalberla et al. (2005) (obtained with the W3NH too 8 ) and fixed to $2.07 \times 10^{20} \mathrm{~cm}^{-2}$ was applied to all the models. Spectral uncertainties are given using

\footnotetext{
${ }_{8}$ http://heasarc.gsfc.nasa.gov/cgi-bin/Tools/w3nh/ w3nh.pl
}

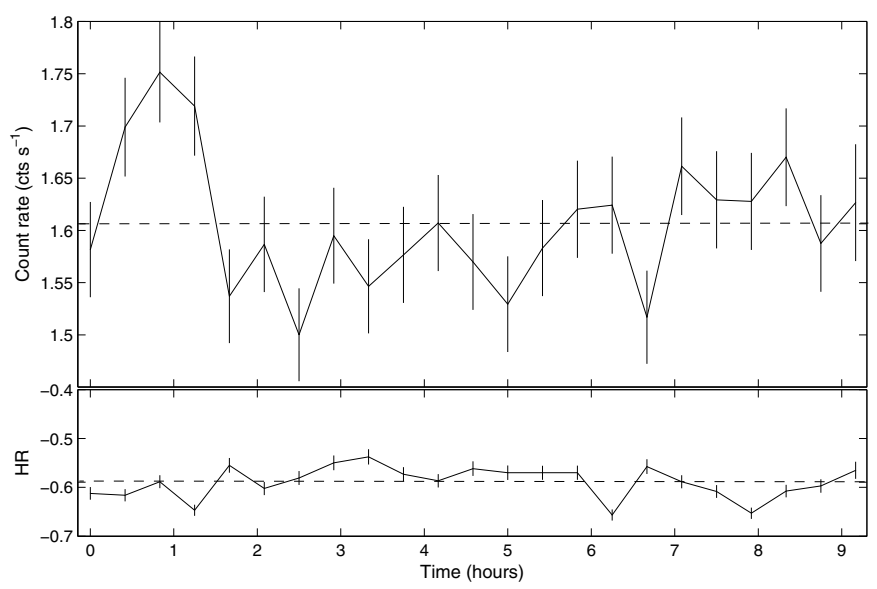

Fig. 3. Upper panel: EPIC-pn light curve of NGC 4278 during the XMM-Newton observation with a time bin size of $1.5 \mathrm{ks}$. Lower panel: hardness ratio. The dashed lines show the average on the count rate and hardness ratio.

$\Delta \chi^{2}$ of 2.71 , corresponding to $90 \%$ confidence for one interesting parameter, and to $95 \%$ confidence for upper limits.

\subsubsection{Chandra spectral analysis}

To obtain a first guess about the models that would be best suitable to the data, we first fit the six pile-up corrected spectra separately. An absorbed power-law model gives an acceptable fit in the six cases with a power-law photon index, $\Gamma$, between 2.3 and 2.8. The intrinsic hydrogen column density has an upper limit of $1.2 \times 10^{21} \mathrm{~cm}^{-2}$. In five of the six observations, the soft $0.5-2 \mathrm{keV}$ band shows residuals around $\sim 1 \mathrm{keV}$ suggesting the possible presence of emission from hot diffuse gas (e.g., Flohic et al. 2006). Figure 4 shows the residuals from the single absorbed power-law fit. We note that the only observation that did not show any evidence for residuals around $\sim 1 \mathrm{keV}$ is the one with the least net source counts (Obs. ID: 4741). The addition of a thermal component (mekal model; Mewe et al. 1985) takes account of these residuals in the soft band and improves the fit quality in the six observations.

Next, we fit the six spectra simultaneously in order to identify the best physical model suitable for the six Chandra spectra and to better constrain the model parameters. For this purpose, we tried three different models:

1. Two power-laws affected by absorption, where the first power-law represents the soft $0.5-2 \mathrm{keV}$ emission from the AGN and the second power-law represents the $2-10 \mathrm{keV}$ compton up-scattered emission by a corona.

2. A power-law affected by intrinsic absorption and a thermal component. The power-law represents the direct continuum emission from the AGN whereas the thermal component takes into account possible emission from unresolved X-ray binaries and/or supernovae remnants.

3. A power-law and a thermal component affected by absorption and an additional power-law component (Guainazzi et al. 2005). This model is similar to model (2) but includes the possibility of Compton-scattered X-ray emission.

Model (1) gives an acceptable fit with a reduced $\chi^{2}$ of 1.38 for 311 d.o.f. However, residuals around $1 \mathrm{keV}$ persisted in the different spectra. We then tried model (2) leaving the intrinsic absorption component, the power-law, and the mekal parameters free for the six spectra. The temperature and the emission 
Table 2. Best fit parameters of a mekal plus an absorbed power-law model for the Chandra data and an absorbed power-law model for the XMM-Newton spectra.

\begin{tabular}{|c|c|c|c|c|c|c|c|c|}
\hline Obs. ID & $\begin{array}{c}\mathrm{Nh}^{a} \\
10^{20} \mathrm{~cm}^{-2}\end{array}$ & $\Gamma$ & $\begin{array}{l}\text { Pl norm. } \\
10^{-4} \text { Photons keV } \mathrm{kem}^{-1} \mathrm{~cm}^{-1}\end{array}$ & $\begin{array}{l}k T^{a} \\
\mathrm{keV}\end{array}$ & $\begin{array}{c}E M^{a b} \\
10^{62} \mathrm{~cm}^{-3}\end{array}$ & $\begin{array}{c}\text { Flux }^{c} \\
10^{-13} \mathrm{erg} \mathrm{s}^{-1}\end{array}$ & $\begin{array}{l}\text { Corr. flux } \\
10^{-13} \mathrm{erg} \mathrm{s}^{-1}\end{array}$ & $\begin{array}{r}\mathrm{Pl}^{d} \\
\%\end{array}$ \\
\hline 4741 & $<6.78$ & $2.13[2.00-2.28]$ & $4.28[3.92-4.71]$ & $0.62[0.58-0.66]$ & $2.64[2.27-2.99]$ & $18.1[17.1-18.8]$ & $18.5[18.2-20.1]$ & 94 \\
\hline 7077 & & $2.26[2.16-2.39]$ & $1.82[1.68-1.99]$ & & & $7.8[7.5-7.9]$ & $8.21[8.0-9.0]$ & 83 \\
\hline 7078 & & $2.34[2.22-2.47]$ & $4.21[3.90-4.61]$ & & & $16.0[14.9-16.2]$ & $16.5[16.1-18.5]$ & 93 \\
\hline 7079 & & $2.38[2.28-2.50]$ & $3.76[3.54-4.07]$ & & & $14.1[13.3-14.3]$ & $15.05[14.7-16.4]$ & 90 \\
\hline 7080 & & $2.02[1.84-2.20]$ & $1.10[0.96-1.26]$ & & & $5.9[5.5-6.1]$ & $6.0[5.7-6.8]$ & 80 \\
\hline 7081 & & $2.12[2.00-2.25]$ & $1.25[1.14-1.29]$ & & & $6.2[5.9-6.4]$ & $6.4[6.2-7.1]$ & 81 \\
\hline \multicolumn{9}{|c|}{ Reduced $\chi^{2}=0.93$ for 310 d.o.f. } \\
\hline 0205010101 & $3.82[3.05-4.59]$ & $2.05[2.03-2.07]$ & $8.07[7.90-8.24]$ & & & $33.8[32.8-33.2]$ & $34.5[34.2-34.8]$ & 100 \\
\hline
\end{tabular}

Notes. ${ }^{(a)}$ Parameter linked for the different Chandra observations; ${ }^{(b)}$ the emission measure (EM) of the $m e k a l$ model, $E M=\int n_{\mathrm{e}} n_{\mathrm{H}} \mathrm{d} V ;{ }^{(c)}$ fluxes in the $0.5-8 \mathrm{keV}$ energy range; ${ }^{(d)}$ the power-law component fraction to the total corrected flux.

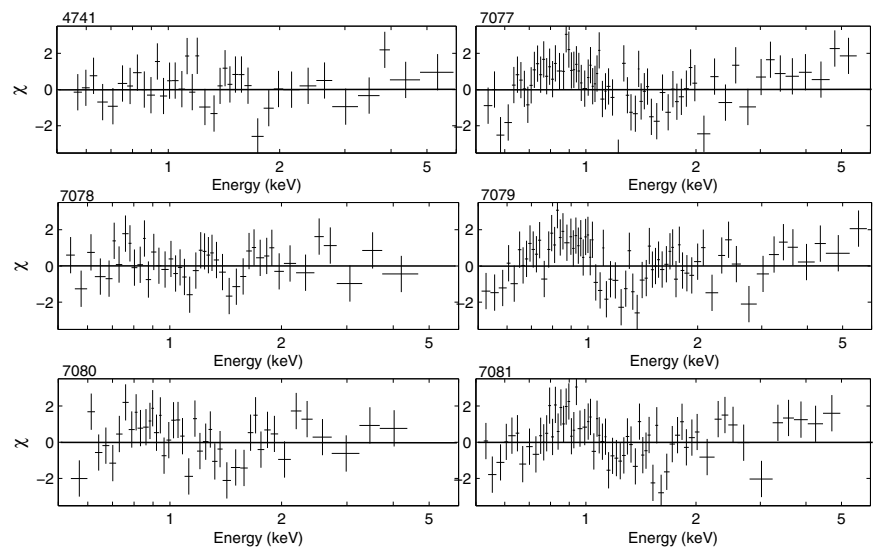

Fig. 4. The residuals in terms of sigma of an absorbed power-law fit to the different Chandra observations. Some features are clearly present in the soft $0.5-2 \mathrm{keV}$ band suggesting emission from diffuse hot gas.

measure (EM) of the thermal component, as well as the hydrogen column density, were consistent within the error bars during the different observations and therefore linked between each other. The fit gives a reduced $\chi^{2}$ of 0.93 for 310 d.o.f. The mean average of the photon index, $\Gamma$, is $2.2_{-0.2}^{+0.1}$. The temperature of the thermal component is $0.62 \pm 0.04 \mathrm{keV}$. The intrinsic hydrogen column density affecting the power-law has an upper limit of $6.78 \times 10^{20} \mathrm{~cm}^{-2}$. According to an F-test the statistical improvement in the fit between model (1) and (2) is highly significant. Finally, we tried model (3) used to fit the spectra of Comptonthin Seyfert 2 galaxies. Similar to model (2), the parameters of the thermal component and the hydrogen column density were linked for the different spectra. We find a reduced $\chi^{2}$ of 0.93 for 309 d.o.f. The absorption hydrogen column density has an upper limit of $8 \times 10^{20} \mathrm{~cm}^{-2}$. The average value of the photon index, $\Gamma$, is $2.3 \pm 0.2$. The temperature of the thermal component is $0.63_{-0.05}^{+0.04} \mathrm{keV}$. The addition of a second power-law did not improve the quality of the fit. The parameters of the thermal model and the power-law model did not change between model (2) and model (3) while the hydrogen column density is better constrained using model (2). Moreover, according to an F-test, the improvement of the fit by adding a power-law component is not significant.
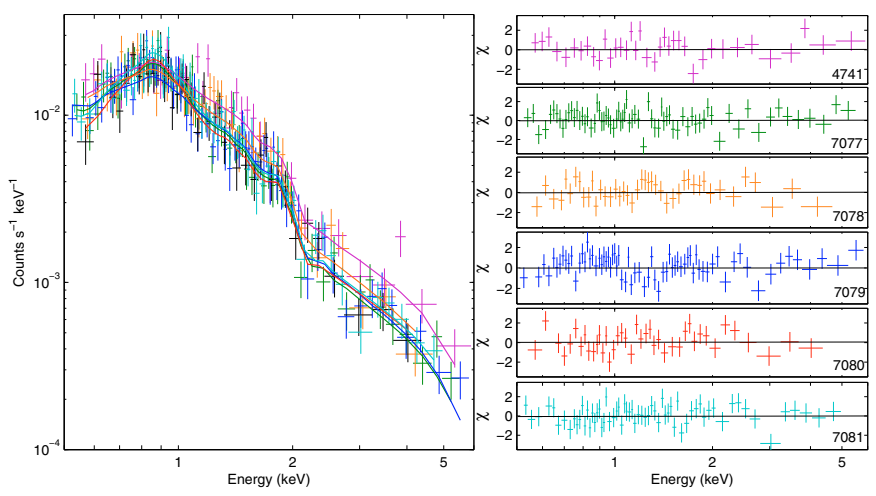

Fig. 5. Left panel: data and simultaneous best-fit model of an absorbed power-law plus mekal to the six Chandra observations. Right panels: the residuals of the fit in terms of sigma. The features in the soft band were accounted for by the thermal component. See the electronic edition of the Journal for a colour version of this figure.

Therefore, we consider model (2), an absorbed power-law and a thermal mekal component, as best suitable for the six Chandra spectra and we show in Fig. 5] the data and the best fit model with the residuals in the right panels. Table 2 gives the parameters of the best fit model, the uncorrected and the corrected $0.5-8 \mathrm{keV}$ flux, as well as the power-law fraction to the total corrected flux.

\subsection{2. $X M M-N e w t o n$ spectral analysis}

The Chandra image of NGC 4278 (obs. ID: 7077) is shown in the right panel of Fig. 2. The central bright source, the NGC 4278 nucleus, is surrounded by a $10^{\prime \prime}$-radius circle. The left panel is the XMM-Newton/MOS1 image with a circle of the same size centered on the source. The lower angular resolution of XMM-Newton compared to Chandra blended the resolved and unresolved point sources, as well as the diffuse emission, in one point source. In order to exclude the contribution from the contaminating sources, the six spectra of the two brightest objects, marked source 1 and source 2 in the Chandra image, obtained in the energy range $0.5-8 \mathrm{keV}$ from each observation were fit simultaneously with an absorbed power-law, assuming that these sources are Low Mass X-ray 
Binaries (LMXBs) Kim \& Fabbiano 2004). We then excluded the two sources and the central object and fit the rest, obtained in the energy range $0.5-8 \mathrm{keV}$, with a combination of a thermal component and a power-law assuming contribution from diffuse emission and unresolved point sources. The point sources and the diffuse emission do not show significant variability between the six Chandra observations and therefore we assumed them to be constant (detailed results on the two point-like sources and the diffuse emission are presented in Appendix B). When fitting the XMM-Newton spectra obtained from the $10^{\prime \prime}$ circle around the point-like source, we included the contribution from all of the above (Source 1, source 2, and the diffuse emission that contribute only to $2 \%$ of the total flux, see Appendix B for more details), the rest is then attributed to the nucleus.

We started the analysis by fitting the pn, MOS1, and MOS2 spectra simultaneously with an absorbed power-law model leaving the normalization free to take account for slight cross calibration uncertainties between the three detectors. The fit is noticeably acceptable with a reduced $\chi^{2}$ of 1.01 for 487 d.o.f. The photon index, $\Gamma$, and the normalization of the power-law are $2.05 \pm 0.02$ and $(7.73 \pm 0.17) \times 10^{-4}$ photons $\mathrm{keV}^{-1} \mathrm{~cm}^{-2} \mathrm{~s}^{-1}$ respectively. The intrinsic hydrogen column density is $3.82 \pm 0.77 \times 10^{20} \mathrm{~cm}^{-2}$. We note that this value is better constrained than the value derived from the Chandra fit. Then, we tried to fit the XMM-Newton spectra with the same models used on the Chandra data. The combination of two absorbed power-laws, model (1), did not give a better fit than one single absorbed power-law with a reduced $\chi^{2}$ of 1.02 for 484 d.o.f. The addition of an unabsorbed thermal component to the absorbed power-law, model (2), gives an acceptable fit with a reduced $\chi^{2}$ of 0.99 for 482 d.o.f. An F-test indicates that the probability for such an improvement (compared to the fit with a single absorbed power law) to occur by chance is 0.25 . Moreover, the emission from the power-law component dominates the total nuclear emission to a $99 \%$ level even at low energies. Therefore, the thermal component is not required by the data and was not kept in the model. Model (3), where an additional absorbed power-law is added to model (2), did not ameliorate the quality of the fit with a reduced $\chi^{2}$ of 1.01 for 479 d.o.f. Therefore, the absorbed power-law model is favored for the XMM-Newton spectra. We give in Table 2 the best fit parameters. Figure 6 shows the data, folded model, and the fit residuals.

No evidence is found for an $\mathrm{Fe} \mathrm{K} \alpha$ emission line at $6.4 \mathrm{keV}$, neither in the pn spectrum nor in the Chandra spectra. The upper limit derived on its equivalent width (EW) is measured from the $X M M$-Newton observation as the spectral resolution of the EPIC$\mathrm{pn}$ is higher than that of the ACIS-S Chandra instrument. By including a Gaussian profile with a width fixed to 0 and $0.3 \mathrm{keV}$ (for a narrow and a broad line respectively) at $6.4 \mathrm{keV}$ we derive upper-limits of $22 \mathrm{eV}$ and $118 \mathrm{eV}$ on the EW's of the narrow and broad components to the $6.4 \mathrm{keV}$ line respectively, which are commonly detected in the spectra of normal Seyferts and quasars (Porquet et al. 2004; Jiménez-Bailón et al. 2005).

\subsection{Optical data}

\subsubsection{XMM-Newton optical/UV monitor}

The optical/UV monitor (OM) detector on board of XMM-Newton observed NGC 4278 for six 2880 s exposures in the $U V M 2$ and $U V W 1$ filters. The central region of NGC 4278 in the UVM2 filter is detected as a point-like source not showing any sign of diffuse emission from the host galaxy.

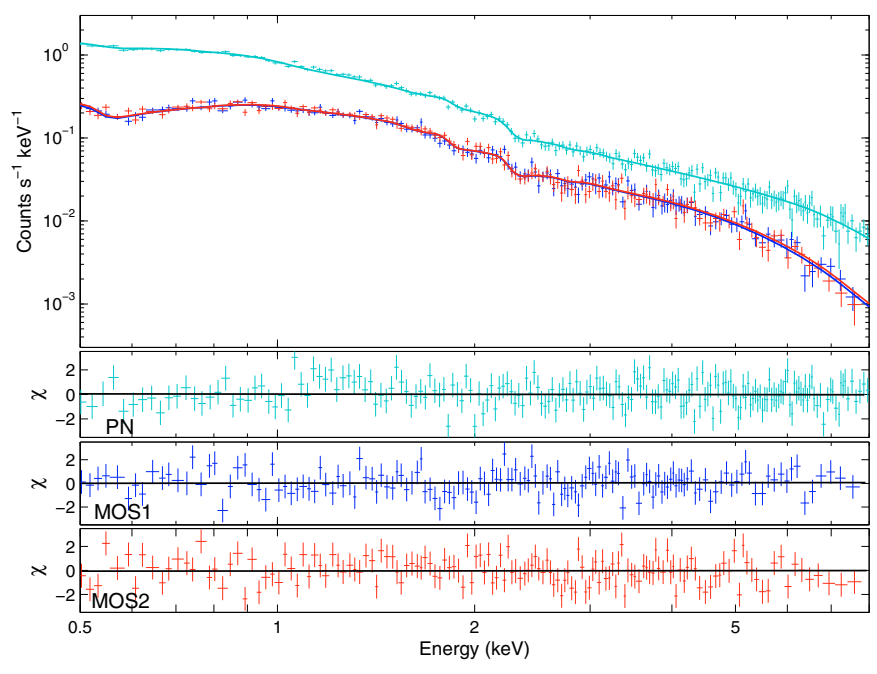

Fig. 6. Upper panel: data and best fit model of an absorbed power-law to the PN, MOS1 and MOS2 (from top to bottom). The three other panels show the residuals of the fit in terms of sigma.

The SAS tool omichain produced an OM source list based on a $3^{\prime \prime}$ aperture and background taken from an aperture with radii $3.7^{\prime \prime}-4.3^{\prime \prime}$. The nuclear corrected count rate in the UVM2 is 0.56 counts $\mathrm{s}^{-1}$ which corresponds to a flux density of $1.18 \times$ $10^{-15} \mathrm{erg} \mathrm{cm}^{-2} \mathrm{~s}^{-1} \AA^{-1}$ (using a count rate to flux conversion factor of $2.103 \times 10^{-15} \mathrm{erg} \mathrm{cm}^{-2} \AA^{-1}$ counts $^{-1}$ ). Cardullo et al. (2009) show the observation of NGC 4278 made with the HST WFPC2/F218 filter (band-pass: 2000-2500 $\AA$, comparable to UVM2 band-pass: 2050-2450 $\AA$ ) in 1994 and in 1995. It is clear in their figures that the dominant source of emission in this specific band-pass is the central source. Therefore, we can consider that the flux calculated from the UVM2 filter corresponds to the nuclear flux. We decided not to use the UVW1 image as it shows strong diffuse emission, most likely from the host galaxy, around the central source.

\subsubsection{HST}

NGC 4278 was observed four times with each of the ACS/WFC F475W and F850LP filters between December 2006 and January 2007. For every filter, we derive the flux for two pointings where the source lies away from the CCD edge. We use the flux derived from the Hubble Legacy Archive (HLA $)^{10}$ DAOPhot tool, originally in counts $\mathrm{s}^{-1}$, and convert it to erg cm $\mathrm{cm}^{-2} \mathrm{~s}^{-1}$ using the conversion equation $F_{\lambda}=C /\left(\varepsilon_{f} \int S_{\lambda} \mathrm{d} \lambda\right)$ where $C$ is the count rate, $\varepsilon_{f}$ is the fraction of the point source energy encircled within $N$ pixels, and $\int S_{\lambda} \mathrm{d} \lambda$ is the total imaging point source sensitivity 11 . We find a flux in the $F 475 \mathrm{~W}$ filter of $6.64 \times 10^{-13} \mathrm{erg} \mathrm{cm}^{-2} \mathrm{~s}^{-1}$ and $8.02 \times 10^{-13} \mathrm{erg} \mathrm{cm}^{-2} \mathrm{~s}^{-1}$ for observations made in December 23, 2006 and January 2, 2007 respectively. The fluxes derived for the $F 850 L P$ filter are $7.45 \times$ $10^{-13} \mathrm{erg} \mathrm{cm}^{-2} \mathrm{~s}^{-1}$ and $8.08 \times 10^{-13} \mathrm{erg} \mathrm{cm}^{-2} \mathrm{~s}^{-1}$ for observations made on December 23, 2006 and January 2, 2007 respectively.

\footnotetext{
9 http://web.archive.org/web/20050322143642/xmm. vilspa.esa.es/sas/documentation/watchout/uvflux.shtml 10 http://hla.stsci.edu/hlaview.html

11 more details can be found at http://www.stsci.edu/hst/acs/documents/handbooks/ cycle18/c09_expcalc3.html
} 
Table 3. Multiwavelength nucleus data for NGC 4278.

\begin{tabular}{|c|c|c|c|c|c|}
\hline $\begin{array}{l}v \\
(\mathrm{~Hz})\end{array}$ & $\begin{array}{c}v L_{v} \\
\left(\operatorname{erg~s}^{-1}\right)\end{array}$ & $\begin{array}{c}\text { Aperture } \\
(\operatorname{arcsec})\end{array}$ & Satellite/filter & Date & Reference \\
\hline $1.93 \times 10^{18}$ & $7.83 \times 10^{39}$ & 3.34 & Chandra/ACIS-S & February 2007 & This work \\
\hline $1.93 \times 10^{18}$ & $5.56 \times 10^{40}$ & 10 & $X M M-N e w t o n / E P I C-p n$ & May 2004 & This work \\
\hline $1.21 \times 10^{17}$ & $1.32 \times 10^{40}$ & 3.34 & Chandra/ACIS-S & February 2007 & This work \\
\hline $1.21 \times 10^{17}$ & $5.96 \times 10^{40}$ & 10 & XMM-Newton/EPIC-pn & May 2004 & This work \\
\hline $1.42 \times 10^{15}$ & $1.05 \times 10^{41}$ & 0.28 & $H S T / \mathrm{F} 218 \mathrm{~W}$ & January 1995 & Cardullo et al. (2009) \\
\hline $1.42 \times 10^{15}$ & $6.75 \times 10^{40}$ & 0.28 & HST/F218W & June 1994 & Cardullo et al. (2009) \\
\hline $1.30 \times 10^{15}$ & $1.21 \times 10^{41}$ & 3 & XMM-Newton/UVM2 & May 2004 & This work \\
\hline $6.32 \times 10^{14}$ & $3.03 \times 10^{40}$ & 0.15 & $H S T / \mathrm{F} 475 \mathrm{~W}$ & January 2007 & This work \\
\hline $6.32 \times 10^{14}$ & $2.51 \times 10^{40}$ & 0.15 & $H S T / \mathrm{F} 475 \mathrm{~W}$ & December 2006 & This work \\
\hline $5.76 \times 10^{14}$ & $1.13 \times 10^{40}$ & 1.26 & HST/F555W & May 1994 & Lauer et al. (2005) \\
\hline $3.65 \times 10^{14}$ & $6.95 \times 10^{39}$ & $<6$ & $H S T / F 814 \mathrm{~W}$ & May 1994 & Capetti et al. (2002) \\
\hline $3.32 \times 10^{14}$ & $2.83 \times 10^{40}$ & 0.15 & $H S T / \mathrm{F} 850 \mathrm{LP}$ & January 2007 & This work \\
\hline $3.32 \times 10^{14}$ & $2.61 \times 10^{40}$ & 0.15 & $H S T / F 850 L P$ & December 2006 & This work \\
\hline $2.40 \times 10^{14}$ & $4.56 \times 10^{42}$ & 8.00 & $1.54 \mathrm{~m}$ Mount Lemmon/J & & Heckman et al. (1983) \\
\hline $1.82 \times 10^{14}$ & $7.77 \times 10^{42}$ & 7.20 & $3.8 \mathrm{~m} \mathrm{UKRIT} / \mathrm{H}$ & & Longmore \& Sharples (1982) \\
\hline $1.36 \times 10^{14}$ & $4.50 \times 10^{42}$ & 7.20 & $3.8 \mathrm{~m} \mathrm{UKRIT/K}$ & & Longmore \& Sharples (1982) \\
\hline $8.69 \times 10^{13}$ & $9.57 \times 10^{41}$ & 8.00 & $1.54 \mathrm{~m}$ Mount Lemmon/L & & Heckman et al. (1983) \\
\hline $2.97 \times 10^{13}$ & $1.15 \times 10^{41}$ & 5.60 & $1.54 \mathrm{~m}$ Mount Lemmon/N & & Heckman et al. (1983) \\
\hline $2.20 \times 10^{10}$ & $5.36 \times 10^{38}$ & $<1$ & VLA & August 2003 & Giroletti et al. (2005) \\
\hline $1.50 \times 10^{10}$ & $4.00 \times 10^{38}$ & 0.15 & VLA & September 1999 & Nagar et al. (2001) \\
\hline $8.40 \times 10^{9}$ & $2.66 \times 10^{38}$ & $(3.2 \times 1.9) \times 10^{-3}$ & VLBA/VLA & August 2000 & Giroletti et al. (2005) \\
\hline $5.00 \times 10^{9}$ & $2.00 \times 10^{38}$ & $(3.2 \times 1.9) \times 10^{-3}$ & VLBA & July 1995 & Giroletti et al. (2005) \\
\hline $5.00 \times 10^{9}$ & $2.25 \times 10^{38}$ & $2.5 \times 10^{-3}$ & VLBA/VLA & August 2000 & Giroletti et al. (2005) \\
\hline $1.67 \times 10^{9}$ & $1.00 \times 10^{38}$ & $5.0 \times 10^{-3}$ & VLBI & May 1979 & Jones et al. (1984) \\
\hline
\end{tabular}

Notes. Infrared, optical and UV luminosities are corrected for galactic extinction.

\section{Spectral energy distribution}

We compile the available photometry data of the nucleus of NGC 4278 from the literature and add the data derived in this work to construct the NGC 4278 SED. In order to minimize any contamination from the host galaxy, high angular resolution is required at all wavelengths. Therefore, only data coming from observations with apertures less than $10^{\prime \prime}$ are used. Table 3 lists the data used to derive the NGC 4278 SED shown in Fig. 7.

In radio wavelengths, and in order to minimize the contamination from the radio jet emission, milli-arcsec data, measured through VLBI techniques, are used when available. Otherwise, VLA observations with sub-arcsec resolution are applied. All of the near-IR (1-3 $\mu \mathrm{m})$ data for NGC 4278 should be considered as upper limits as the resolution of those observations is between $5^{\prime \prime}$ and $10^{\prime \prime}$. Only the 10-20 $\mu \mathrm{m}$ mid-IR point could be considered as less affected by emission from normal stellar populations and may represent to some extent the nuclear emission (thermal emission from dust grain can not be ruled out). Optical and UV data are obtained from Hubble Space Telescope (HST) observations where $1^{\prime \prime}$ apertures are used. One UV data point coming from the XMM-Newton OM telescope is used (see Sect. 3.3.1). X-ray data, measured in this paper, and derived from Chandra and XMM-Newton observations are used.

The Galactic extinction toward NGC 4278 is 0.11 mag (de Vaucouleurs et al. 1991). The same value is derived using the Galactic hydrogen column density and applying the equations $E(B-V)=\mathrm{NH} /\left(5.8 \times 10^{21}\right) \mathrm{mag}$ and $A_{V} / E(B-V)=3.1$. Internal extinction is reported in Ho et al. (1997) to be 0 according to a Balmer decrement $\mathrm{H} \alpha / \mathrm{H} \beta=2.50$ leading to $E(B-V)=0$. Infrared, optical and UV data are de-reddened according to

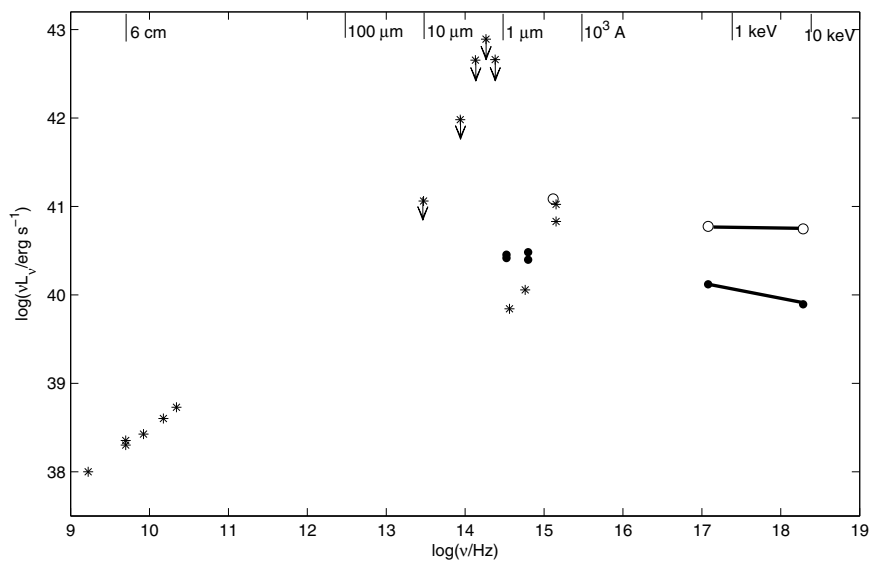

Fig. 7. The spectral energy distribution of NGC 4278. Stars derive from non simultaneous radio to UV observations. The open circles are the simultaneous XMM-Newton UV and X-ray fluxes. The dots represent the contemporary HST/ACS optical and the Chandra (obs. ID: 7081) X-ray fluxes.

these extinctions and adopting the Galactic extinction curve of Cardelli et al. (1989).

The radio slope between 5 and $22 \mathrm{GHz}$ is somewhat inverted $(\alpha \sim-0.6)$, typical for LINERsI/LLAGN (Ho 1999). Radio loudness is quantified using the radio to $\mathrm{X}$-ray ratio defined as $R_{\mathrm{X}}=v L_{v}(6 \mathrm{~cm}) / L_{\mathrm{X}}$, where $L_{\mathrm{X}}$ is the luminosity in the $2-10 \mathrm{keV}$ band (Terashima \& Wilson 2003). Following this criterion, NGC 4278 is considered as a radio-loud LINER with $R_{\mathrm{X}}$ 
ranging between $2.4 \times 10^{-2}$ and $3.5 \times 10^{-3}$ where $R_{\mathrm{X}}=3.162 \times$ $10^{-5}$ is the boundary for radio loud systems. There is a tendency for a maximum in the SED at mid-IR wavelengths where stellar population emission is less effective, although thermal emission from dust grains can not be ruled out. The UVM2 flux derived from the OM telescope is used to derive an optical to $\mathrm{X}$-ray slope $\alpha_{\mathrm{ox}}$ and a radio loudness parameter $R_{\mathrm{UV}}$ where $\alpha_{\mathrm{Ox}}=$ $0.384 \log \left(L_{2 \mathrm{keV}} / L_{2500} \AA\right)$ and $R_{\mathrm{UV}}=\log \left(L_{v}(6 \mathrm{~cm}) / L_{v}(2500) \AA\right)$. These two parameters are later used for comparison purposes with LINER and normal Seyfert galaxies. We derive a $2 \mathrm{keV}$ luminosity $v L_{v}(2 \mathrm{keV}) \approx 4 \times 10^{40} \mathrm{erg} \mathrm{s}^{-1}$ from the unfolded model of the XMM-Newton pn spectrum which gives an $\alpha_{\text {ox }}$ of -1.2 . We emphasize that this value of $\alpha_{\text {ox }}$ is determined, for the first time for NGC 4278, from simultaneous UV and X-ray observations. $R_{\mathrm{UV}}$ is about 2.7 and the $2-10 \mathrm{keV}, \alpha_{\mathrm{x}}(\Gamma-1)$, spectral index is $\sim 1.2$.

\section{Discussion}

\subsection{X-ray properties}

The NGC 4278 Chandra observations are well fitted with a combination of an absorbed power-law and a thermal component. The temperature of the thermal model is $0.62 \pm 0.04 \mathrm{keV}$ for the different observations. The power-law photon index, $\Gamma$, has an average of $2.2_{-0.2}^{+0.1}$. As for the XMM-Newton observation, an absorbed power-law alone is sufficient to fit the spectra well with a $\Gamma$ of $\sim 2.1$. The intrinsic hydrogen column density affecting the power-law is of the order of $10^{20} \mathrm{~cm}^{-2}$ in both cases. The fluxes derived for the Chandra observations vary between $5.9 \times 10^{-13}$ and $18.1 \times 10^{-13} \mathrm{erg} \mathrm{cm}^{-2} \mathrm{~s}^{-1}$ while the flux measured for the XMM-Newton observation is $33.8 \times$ $10^{-13} \mathrm{erg} \mathrm{cm}^{-2} \mathrm{~s}^{-1}$. Terashima \& Wilson (2003) studied a sample of LLAGN nuclei, that display a compact radio source, observed with Chandra. The authors found that the $0.5-8 \mathrm{keV}$ spectra of all the sources could be explained with an absorbed power-law with most of the objects having a hard X-ray spectrum with a photon index $<2$, including NGC 4278. Our analysis of the Chandra and XMM-Newton observations showed a somewhat softer $0.5-8 \mathrm{keV}$ spectrum with $\Gamma$ greater than 2 . The NGC 4278 observation treated by Terashima \& Wilson (2003) was affected by pile-up at a level greater than $10 \%$ and at such a high level, the pile-up model of Davis (2001), used by Terashima \& Wilson (2003) to correct for pile-up, is misleading. González-Martín et al. (2009) studied a sample of 82 LINERs observed with Chandra and/or XMM-Newton and fit the spectra, in most of the cases, with a combination of powerlaw and a thermal component. The authors find a median value for the photon index, $\Gamma$, of $2.11 \pm 0.52$ and a thermal component temperature of $0.54 \pm 0.30 \mathrm{keV}$, compatible with the values we find for NGC 4278. The authors find a bimodal distribution for the 2-10 keV corrected luminosities they derived for their sample centered at $L(2-10 \mathrm{keV})=10^{39} \mathrm{erg} \mathrm{s}^{-1}$ and $L(2-10 \mathrm{keV})=10^{41} \mathrm{erg} \mathrm{s}^{-1}$. At a lower flux, NGC 4278 lies in the low luminosity part of the group with a $2-8 \mathrm{keV}$ luminosity of $8 \times 10^{39} \mathrm{erg} \mathrm{s}^{-1}$ while at higher flux, NGC 4278 moves to the high luminosity group with a $2-8 \mathrm{keV}$ luminosity of $6 \times$ $10^{40} \mathrm{erg} \mathrm{s}^{-1}$. Additionally, González-Martín et al. (2009) studied one Chandra observation (obs. ID: 7077) of NGC 4278 and found a photon index of $2.6_{-0.3}^{+0.1}$ and a thermal temperature of $0.5_{-0.1}^{+0.2} \mathrm{keV}$ comparable to the values we derive for the same observation. However, they find an observed $0.5-10 \mathrm{keV}$ flux of $\sim 4 \times 10^{-13} \mathrm{erg} \mathrm{s}^{-1} \mathrm{~cm}^{-2}$ which is half the value we derive for the same observation. However, González-Martín et al. (2009)

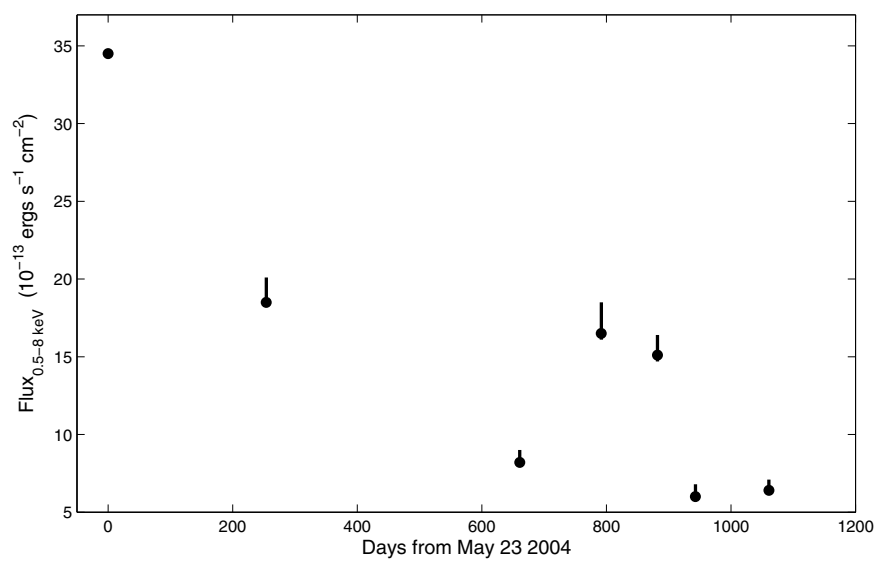

Fig. 8. The long term X-ray variability of NGC 4278.

did not correct for the $\sim 8 \%$ pile-up fraction that could explain the discrepancy between the two derived flux values as pile-up tempt to underestimate flux measurements (see Appendix A).

\subsection{Variability}

At radio wavelengths, NGC 4278 does not show significant inter-year variability at 2 or $3.6 \mathrm{~cm}$ (Nagar et al. 2002). At $6 \mathrm{~cm}$, Giroletti et al. (2005) discovered modest variability on yearly time-scales with the flux decreasing by a factor of $10 \%$ between 1995 and 2000. At UV wavelengths, Cardullo et al. (2009) discovered that NGC 4278 is variable on months timescales showing that its flux increased $\sim 2$ times between two different HST observations. This is in agreement with Maoz et al. (2005) who discovered variability in 12 of 16 LINERsI/LLAGN objects observed with the HST ACS/HRC F250W and F330W filters. Nine of the 12 galaxies that show UV variability in their sample have detected radio cores suggesting a connection between UV variability and the presence of radio cores. NGC 4278 enforces this correspondence as it shows a luminous radio core as well as significant UV variability. Additionally, we detect optical nuclear variability on years time-scale as the fluxes we derive from $H S T$ ACS $F 475 W$ and F850LP filters are 2 to 4 times higher than optical fluxes derived from HST WFPC2 F555W and $F 814 W$ filters (we do not discard optical variability on shorter time-scales but no intra-year optical observations were ever made for NGC 4278). In X-rays, NGC 4278 show no significant short time-scale (hours to days) variability during the different Chandra observations with the "excess variance", $\sigma_{\text {rms }}^{2}$, never exceeding 0.01 , with an error $<15 \%$, which means that NGC 4278 does not follow the same trend as Seyfert galaxies showing stronger variability with decreasing luminosity (Nandra et al. 1997). On the other hand, during the XMM-Newton observation, NGC 4278 exhibits short-time scale variability with the flux increasing by a factor of $10 \%$ on a $1 \mathrm{~h}$ period. Moreover, a significant monthly time-scale variability is observed between the different Chandra observations and between Chandra and $X M M$-Newton observations (Fig. 8) where the flux increased by a factor of $\sim 3$ on a few months time-scale and by a factor of 5 between the faintest and the brightest observation separated by $\sim 3$ years. Ptak et al. (1998) suggested that the lack of variability on short $(<1$ day) time-scales and the detection of variability on longer time-scales could be due to the fact that the regions responsible for the X-ray emission are larger in LINERs and LLAGN than in typical Seyfert galaxies. One possibility is that the X-ray emission originates from an optically 
thin geometrically thick flow (RIAF) rather than from a geometrically thin accretion disk.

This scenario could be present during the different Chandra observations corresponding to the lowest flux level. The lack of variability on time-scales of hours to days found during the Chandra observation could be compatible with a large emission region, such as an extended RIAF emission. However, in AGN an anti-correlation exists between the $2-10 \mathrm{keV} X$-ray variability and the mass of the black hole ( $\mathrm{Lu} \mathrm{\&} \mathrm{Yu} \mathrm{2001)} \mathrm{with} \mathrm{some}$ exceptions whenever the accretion rate is high (e.g., Reeves et al. 2002). Hence, the lack of variability during the Chandra observations in NGC 4278 is consistent with the high black hole mass of $3 \times 10^{8} M_{\odot}$ (Wang \& Zhang 2003; Chiaberge et al. 2005) and the low accretion rate.

The short time-scale variability $(t \sim 1.5 \mathrm{~h})$, observed during the XMM-Newton observation (where the X-ray flux level was highest), could be explained by a disturbance in the (extended) RIAF or by the presence of a more compact region, e.g., an accretion disk truncated at a lower radius. In the context of X-ray fluctuations in a RIAF scenario, Manmoto et al. (1996) and Takeuchi \& Mineshige (1997) were able to explain the Xray shots seen in Cygnus X-1 light curves (Negoro et al. 1994) as due to a disturbance in an extended RIAF. However, contrarily to Cygnus X-1 that switches from a soft state to a hard state just after the peak, NGC 4278 remains in a soft state during the whole observation (including the variation). Additionally, if the variation in the case of the XMM-Newton observation is the result of a disturbance in the accretion flow, the maximum radius can be determined from the time duration of the variation from the free-fall time-scale (Manmoto et al. 1996):

$T(f f) \approx 4 \times\left(\frac{r}{10^{3} r_{\mathrm{g}}}\right)^{3 / 2}\left(\frac{M}{10 M_{\odot}}\right) s$

where $M$ is the $\mathrm{BH}$ mass, $T(f f)$ is the free-fall time (the duration of the shot), $r$ is the transition radius, and $r_{\mathrm{g}}$ is the gravitational radius, $G M / c^{2}$. This yields to a transition radius of $r \sim 1.3 r_{\mathrm{g}}$ between the RIAF and the classical thin disk. Therefore a disturbance in an extended RIAF region appears unlikely to explain the short time-scale variation observed from NGC 4278, although further works on variability from RIAF are required to establish any definitive conclusion. Therefore, the presence of a more compact region during the XMM-Newton observation, (i.e. the accretion disk is similar to that observed in low luminosity Seyfert 1 galaxies or most probably truncated at a lower radius than that during the Chandra observations) appears to be a more viable explanation for this short time-scale variability.

\subsection{NGC 4278 SED comparison to other LLAGN}

We now turn to examine whether the X-ray continuum emission of NGC 4278 originates from a classical optically thick geometrically thin accretion disk or from a RIAF structure forming around a black hole $(\mathrm{BH})$ whenever the radiative efficiency is very low. The bolometric luminosity $L_{\mathrm{bol}}$ is estimated by integrating the SED from radio to X-ray data, neglecting upper limits such as points in the IR. As mentioned in Sect. 5.2, NGC 4278 as most of LINERsI/LLAGN, show a high degree of variability on months timescales. Hence, two bolometric luminosities were derived where the highest X-ray flux (XMM-Newton flux) is used to derive the highest $L_{\text {bol }}$ and the lowest $X$-ray flux (Chandra obs. ID 7080 flux) is used to calculate the lowest $L_{\mathrm{bol}}$. This gives $L_{\text {bol }}$ between $2.21 \times 10^{41} \mathrm{erg} \mathrm{s}^{-1}$ (low state) and $2.88 \times 10^{41} \mathrm{erg} \mathrm{s}^{-1}$ (high state) which leads to an Eddington ratio $L_{\text {bol }} / L_{\text {Edd }}$ between $5.7 \times 10^{-6}$ and $7.4 \times 10^{-6}$. Similar $L_{\mathrm{bol}} / L_{\text {Edd }}$ value were derived for the LINER galaxy M 81 (Pian et al. 2010). Two possibilities emerge to explain the faint luminosities in both the low and high state; accretion is occurring at sub-Eddington rate and/or the accretion is radiatively inefficient.

Maoz (2007), when studying a sample of 13 nearby galaxies with LINER nuclei, argued that their SED do resemble the SEDs of normal Seyfert galaxies, and hence the mode of accretion, "geometrically thin optically thick accretion flow", and the X-ray continuum, "the result of inverse compton scattering of UV/soft X-ray in a hot optically thin plasma in a corona", are also similar. The author based his arguments on two parameters: the optical to X-ray, $\alpha_{\mathrm{ox}}$, slope (calculated using the $2500 \AA$ flux), and the radio loudness, $R_{\mathrm{UV}}$, (also based on the $2500 \AA$ flux). He showed that the $\alpha_{\text {ox }}$ values derived for his sample overlap with the values derived for a sample of broad-lined AGN (Steffen et al. 2006) and demonstrated that even though LINERsI/LLAGN are louder by a factor of $~ 100$ than highluminosity quasars, most of the LINERs are considered radio quiet compared to their $2500 \AA$ flux. NGC $4278 \alpha_{\text {ox }}$ is -1.2 (see Sect. 4), comparable to the values derived for Maoz (2007) sample of LLAGN and it follows the extension to low luminosities of the relation between the $\alpha_{\text {ox }}$ and the $2500 \AA$ luminosity derived for a sample of Seyfert galaxies (Steffen et al. 2006). Moreover, NGC $4278 R_{\mathrm{UV}}$ is 2.7 , thus it stands in the radio quiet branch of the relation between $R_{\mathrm{UV}}$ and the $2500 \AA$ luminosity for a sample of luminous AGN (Sikora et al. 2007; Maoz 2007). We note that our results may be slightly biased by the fact that the $2500 \AA$ luminosity we derive for NGC 4278 comes from the UVM2 XMM-Newton optical monitor telescope where neither the peak $\lambda$ nor the effective $\lambda$ are as close as the HST ACS/HRC F250W filter to $2500 \AA$ used by Maoz (2007).

A RIAF model has been suggested as a solution for the low luminosity observed in a number of LINERsI/LLAGN; M 81 and NGC 4579 (Quataert et al. 1999), NGC 4258 (Gammie et al. 1999), NGC 3998 (Ptak et al. 2004), NGC 1097 (Nemmen et al. 2006), and, more recently, for a sample of 24 LINER sources (Nemmen et al. 2010). Authors of these papers emphasize the fact that SEDs of LLAGN in general are in fact different than normal Seyfert SEDs. They differ in a number of ways, mainly, the lack of the canonical "big blue bump" at UV wavelengths, observed in almost all of the luminous galaxies, which is associated with thermal emission from a geometrically thin, optically thick accretion disk ( $\mathrm{Ho}$ 2005, 2002). Instead, a maximum in the SED is peaking in the mid-IR. The presence of radio jets is a common feature in LLAGN as for they tend to be radio loud systems. Additionally, the lack of a broad $\mathrm{Fe} \mathrm{K} \alpha$ emission line at $6.4 \mathrm{keV}$ in most of the LLAGN studied up until now, originating from a standard disk in typical luminous AGN, might suggest the truncation of this structure at a radius much larger than the innermost stable circular orbit (ISCO) where a radiatively inefficient accretion flow (RIAF) structure forms in the inner part. We note that the last argument, lack of a broad Fe K line, used to point at the existence of a RIAF in LINERs is not decisive since a small fraction, $25-30 \%$, of Seyfert galaxies exhibit broadened emission in the iron $K$ band (Nandra et al. 2007).

The absence of a narrow Fe $\mathrm{K} \alpha$ emission line at $6.4 \mathrm{keV}$ with a $22 \mathrm{eV}$ upper limit on its $\mathrm{EW}$ is in agreement with the possibility of a RIAF presence at the center of NGC 4278. However, the large upper limit of $118 \mathrm{eV}$ on the EW of a broad line at $6.4 \mathrm{keV}$ is not discriminative. In order to examine more accurately the possibility of a RIAF structure and to identify the accretion mode occurring at the nucleus of NGC 4278 we 


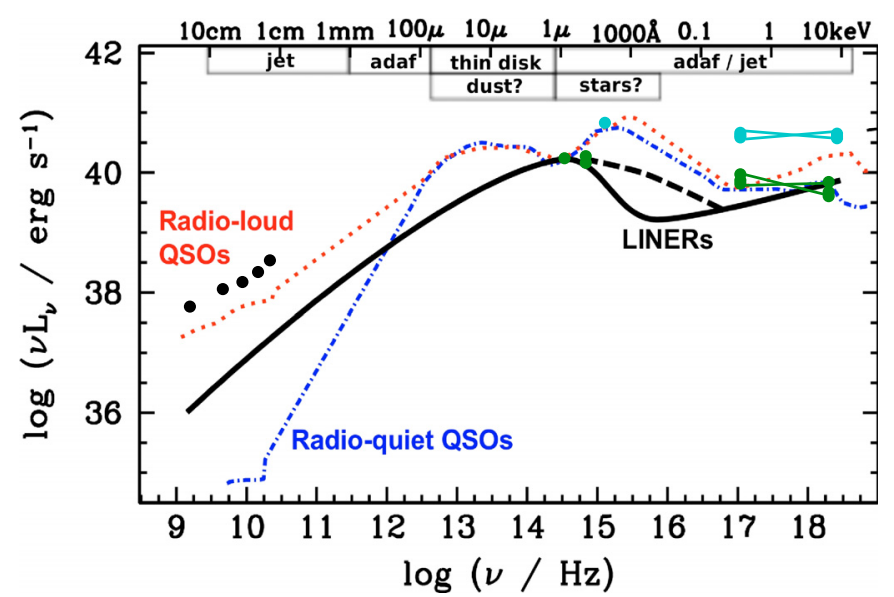

Fig. 9. NGC 4278 SED: black dots represent the radio data. Green dots represent the contemporary HST/ACS and the Chandra 7081 observation fluxes. The light-blue dots correspond to the simultaneous $X M M-N e w t o n$ OM and pn fluxes. The average SED of a sample of LINERs is shown as solid black line (figure adopted from Nemmen et al. 2010). The dashed line represents the effect of extinction correction. For comparison, the average SED of Elvis et al. (1994) for radioloud (dotted line) and radio-quiet (dot-dashed line) quasars. All SEDs are normalized to $1 \mu \mathrm{m}$.

plotted in Fig. 9 (adopted from Nemmen et al. 2010) fluxes derived for NGC 4278 from simultaneous or close-by observations. The black solid line represents the average SED of a sample of LINER nuclei (the dashed black line represents the effect of extinction), the dotted red line and the dot-dashed blue line represents the average SED of Elvis et al. (1994) sample of radio-loud and radio-quiet quasars, respectively. All SEDs are normalized to $1 \mu \mathrm{m}$. Light-blue dots represent the simultaneous XMM-Newton UVM2 flux at UV wavelengths and the EPIC-pn fluxes at X-rays. The green dots represent the ACS/WFC optical data and the Chandra obs. ID 7081 X-ray fluxes. The EPICpn and the OM observations were taken simultaneously and the time difference between the Chandra and the HST data is less than two months and therefore assumed simultaneous as large optical and X-ray variability is observed only on time-scales of several months (see Sect. 5.2). Black dots represent the radio data and are considered constant on time-scale of several years.

If we compare the NGC 4278 SED during the low Chandra $\mathrm{X}$-ray flux (black and green dots combined) to the other SEDs shown in Fig. 9, we can consider NGC 4278 as a radio loud LINER galaxy. The radio emission is more important than the one observed in radio loud quasars, plus, optical and X-ray luminosities correspond to the luminosities derived for the sample of LINERs. On the other hand, the NGC 4278 SED during the $X M M$-Newton observation, where the X-ray flux is the highest (black and light-blue dots combined) seems to be similar to the SEDs of a small number of low luminosity Seyfert 1 galaxies, in which the UV to X-ray slope is almost flat (Papadakis et al. 2008), where the authors showed that the accretion mode in low luminosity Seyfert 1 galaxies $\left(L_{\mathrm{X}} / L_{\mathrm{Edd}}<10^{-4}\right)$ resemble the accretion mode detected in luminous AGN.

As NGC 4278 shows a high degree of variability on months time-scale fluctuating apparently between a low-state SED (black and green dots) and a high-state SED (black and lightblue dots) where short time-scale variability is detected, we consider that the emission mechanism responsible for the bulk of energy from radio to X-rays during the low state is jet/RIAF dominated; a thin accretion disk most probably truncated but at a lower radius than that during the low-state level would be responsible for the optical to X-ray emission during the high-state SED.

\section{Summary and conclusion}

We have studied in detail seven X-ray observations for NGC 4278, six ACIS-S Chandra observations plus one XMM-Newton observation. The observations covered a three year period allowing an extensive variability study. No short time-scale (minutes to hours) variability is detected during the Chandra X-ray observations, while during the XMM-Newton observation, where the highest flux level is observed, the flux increases by a factor of $10 \%$ in a $1 \mathrm{~h}$ period. A significant months time-scale variability is observed between the different Chandra observations and between Chandra and XMM-Newton observations where the flux increased by a factor of $\sim 3$ on a few months time-scale and by a factor of 5 between the faintest and the brightest observations separated by $\sim 3$ years. We checked the validity of increasing variability with decreasing luminosity detected in Seyfert galaxies (Nandra et al. 1997), NGC 4278, like most of LINER sources, does not follow the same trend (Ptak et al. 1998). However, based on the anti-correlation between variability and the mass of the black hole with some exceptions whenever the accretion rate is high (Reeves et al. 2002), no short time-scale variation is expected in NGC 4278 as it harbors a $\sim 3 \times 10^{8} M_{\odot}$ black hole and accretes at low rate. However, the short-time scale variability detected during the XMM-Newton observation might suggest a change in the central engine of NGC 4278 at that point. No variability is detected in the hardness ratio during the observations being soft in all cases.

The best spectral fit found for the six Chandra observations is an absorbed power law plus a thermal component. The intrinsic hydrogen column density affecting the power law has an upper limit of $6.7 \times 10^{20} \mathrm{~cm}^{-2}$, while the average photon index $\Gamma$ is $2.2_{-0.2}^{+0.1}$. The temperature of the thermal component is $0.63_{-0.04}^{+0.05} \mathrm{keV}$. Because of the XMM-Newton moderate angular resolution compared to Chandra, we subtracted, in a $10^{\prime \prime}$ radius, the contribution from X-ray binaries and diffuse emission calculated from the Chandra observations. We find that the powerlaw emission is dominant over the whole $0.5-8 \mathrm{keV}$ band and therefore no thermal component is needed. The hydrogen column density is $(3.8 \pm 0.8) \times 10^{20} \mathrm{~cm}^{-2}$ affecting a power law with a photon index $\Gamma$ of 2.1. No Fe $\mathrm{K} \alpha$ emission line at $6.4 \mathrm{keV}$ is detected with a $22 \mathrm{eV}$ upper limit on its equivalent width.

We measured a UV flux of about $1.2 \times$ $10^{-15} \mathrm{erg} \mathrm{\textrm {cm } ^ { - 2 }} \mathrm{s}^{-1} \AA^{-1}$ from the XMM-Newton optical monitor observation of NGC 4278. The advantage of such a measurement is the simultaneity with the X-ray observation crucial for comparing such sources with normal galaxies as NGC 4278 shows significant variability on months time-scales. We calculated optical fluxes coming from HST ACS observations in two different filters $(F 475 \mathrm{~W}$ and $F 850 L P)$. These fluxes are 2 to 4 times higher than optical fluxes, coming from the $H S T$ WFPC2 $F 555 W$ and $F 814 W$ filters, observed $\sim 12$ years earlier. Our optical fluxes are contemporary to the Chandra fluxes coming from the 7081 observation as the two observations are separated by less than two months.

In order to determine the origin of the emission mechanism responsible for the bulk of energy from radio to X-rays and to assess the geometry of the central engine in NGC 4278 we plotted the SED derived from simultaneous observations 
$($ radid 12 $+\mathrm{UV}+\mathrm{X}$-rays and radio+optical $+\mathrm{X}$-rays) with the SED of a sample of LINER sources (Eracleous et al. 2010) along with radio-quiet and radio-loud quasars (Fig. 8, adopted from Nemmen et al. 2010). As NGC 4278 appears to fluctuate in optical, UV and X-rays on month-timescales, it appears that NGC 4278 SED is consistent with LINER SEDs when the X-ray emission is weak and therefore we suppose a jet/RIAF origin for the radio to X-ray bulk of energy. On the other hand, when the $\mathrm{X}$-ray emission is important, the NGC 4278 SED is similar to the SED of low luminosity Seyfert galaxies and a thin accretion disk is responsible for the bulk of energy from UV to X-rays. Hence, we consider NGC 4278 to alternate between LINER-like and Seyfert-like nuclear activity depending on the strength of its $\mathrm{X}$-ray emission.

Acknowledgements. This research has made use of the data obtained from the Chandra Data Archive and the Chandra Source Catalog, and software provided by the Chandra X-ray Center (CXC) in the application packages CIAO and Chips. This work is based on observations with XMM-Newton, an ESA science mission with instruments and contributions directly funded by ESA Member States and the USA (NASA). This research has made use of the SIMBAD database, operated at CDS, Strasbourg, France. This research has made use of the NASA/IPAC Extragalactic Database (NED) which is operated by the Jet Propulsion Laboratory, California Institute of Technology, under contract with the National Aeronautics and Space Administration. G.Y. would like to thank Patrick Broos for helpful comments on the use of the AE package. The authors would also like to thank the referee for fruitful comments that improved the quality of the manuscript.

\section{Appendix A: Going through pile-up}

When a source is sufficiently bright that two or more photons are incident on the same CCD pixel in a single CCD frame time, the energy of these photons will be added and the observation suffers photon pile-up. Low energy photons will migrate to high energies creating fake high energy events. Furthermore, if the added energy of the photons are greater than the on-board spacecraft threshold, the photon will be rejected by the spacecraft software. Accordingly, piled-up observations will suffer a decrease in the total observed count rate and the spectral shape of the source will be distorted. Although there is no simple way to overcome pile-up, two methods have proved effective. The statistical method of Davis (2001) reconstruct piled events in a spectrum by applying a non-linear integral equation that will connect X-ray source spectra to CCD instrument spectra that takes into account the possibility of photon pile-up. A second more secure approach is to discard spatially and spectrally distorted piled events from the core of the PSF, and stay with non-piled events in the wings of the PSF (Broos et al.1998). This method also reconstruct temporal features and is applicable to sources with any degree of pile-up fractions.

Pile-up could be estimated using the count rate landing in a $3 \times 3$ pixel island and the CCD readout frame time. A simple converter from count rate to pile-up fraction at the on-axis position is written by Dr. M. Tsujimoto and is provided as a $\mathrm{C} \operatorname{code}^{13}$. Unfortunately, we found that all of the seven Chandra observations are affected by pile-up. To overcome this issue, we

\footnotetext{
12 We consider the radio flux constant, and include it in the simultaneous observations since it does not show significant variability on a several years time-scale (see Sect. 5.2).

13 The code, $c r 2 p f . c$, can be found at http://www.astro.isas. jaxa.jp/ tsujimot/arfcorr.html
}

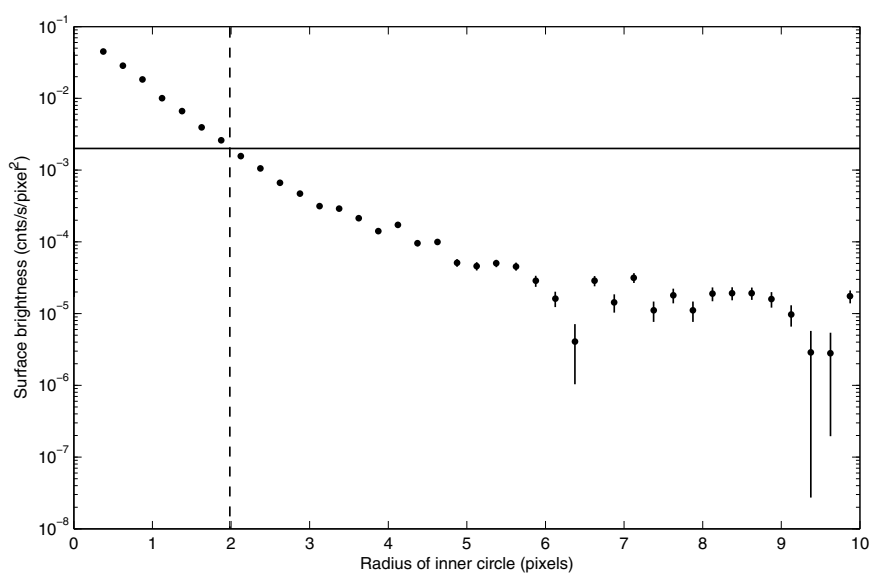

Fig. A.1. Surface brightness profile of NGC 4278 during the observation 7078. The solid horizontal line mark the surface brightness value that corresponds to a $\sim 2 \%$ pile-up fraction. The vertical dashed line shows the radius of the inner circle that has to be discarded.

decided to follow the method prepared by Getman et al. (2005) ${ }^{14}$. The idea is to exclude the piled events from the center of the PSF using the acis_extract (AE) too 15.

We used AE tools to sample the PSF between radii $r_{\text {in }}$ and $r_{\text {out }}$, the latter fixed to $99 \%$ of the PSF, $r_{\text {in }}$ varies between excluding $0 \%$ of the PSF to excluding $90 \%$ of the PSF in steps of $5 \%$, whereas a step of $1 \%$ is used to sample the PSF between $90 \%$ and $95 \%$. Those annuli were then converted from PSF fraction to pixels. The idea is to find the smallest $r_{\text {in }}$ that includes the greatest number of counts for later spectral analysis without reducing the source flux due to pile-up.

Since pile-up is related to the surface brightness of the incident photon distribution, we created the radial profile of annuli regions using the dmextract tool as described in CIAO 16 , and calculated the surface brightness of every extracted annulus. We discarded the cores giving a surface brightness greater than $1.2 \times$ $10^{-3}$ counts s $^{-1}$ pixel $^{-1}$ minimizing the pile-up fraction to $2 \%$. Figure A.1 shows the surface brightness profile of the observation 7078 with the black horizontal line marking the $2 \%$ level of pile-up. Figure A.2 represents the flux profile of the diminishing annuli with the black vertical line marking the $2 \%$ level of pile-up. We notice an increase in the flux when the pile-up fraction decreases and then reaches a plateau after discarding the piled-up core. Table A.1 gives the parameters of the Chandra observations corrected for pile-up.

Considering the small exposure time of the observation 398 that led to a small number of counts, we decided to discard an inner circle sustaining 5\% pile-up fraction. This leaves us with $20 \%$ of the PSF. We show in Fig. A.3 that the photon index is increasing when we go away from the PSF center but the values remain within the error bars. We decided not to include this observation when performing detailed spectral analysis to avoid any misleading results that could generate un-trustful conclusions.

\footnotetext{
${ }_{14}$ More information can be found at http://www.astro.psu.edu/ xray/docs/TARA/ae_users_guide/pileup.txt

15 http://www.astro.psu.edu/xray/docs/TARA/ae_users_ guide.html

${ }^{16}$ Details on how to obtain a radial profile can be found at http:// cxc.harvard.edu/ciao/threads/radial_profile/index.py. html
} 
Table A.1. Pile-up analysis of the Chandra observations.

\begin{tabular}{cccccccc}
\hline \hline OBS. ID & $\begin{array}{c}\text { Exposure } \\
\text { ks }\end{array}$ & Pile-up $\%$ & $\begin{array}{c}r_{\text {in }} \\
\text { Pixels }\end{array}$ & $\begin{array}{c}r_{\text {out }} \\
\text { Pixels }\end{array}$ & Net counts & Extracted PSF fraction & $\begin{array}{c}\text { Corrected count rate } \\
\text { Counts s }^{-1}\end{array}$ \\
\hline $398^{a}$ & 1.4 & 11.5 & 1.45 & 2.42 & 87 & 0.2 & 0.26 \\
4741 & 37 & 20 & 1.90 & 3.88 & 1112 & 0.1 & 0.25 \\
7077 & 110.5 & 7.6 & 1.60 & 4.17 & 2446 & 0.15 & 0.12 \\
7078 & 51 & 14.5 & 1.98 & 3.24 & 1272 & 0.1 & 0.19 \\
7079 & 105 & 13.2 & 2.00 & 3.30 & 2477 & 0.1 & 0.19 \\
7080 & 55 & 5.2 & 1.43 & 3.29 & 1202 & 0.2 & 0.09 \\
7081 & 110 & 5.6 & 1.43 & 3.34 & 2679 & 0.2 & 0.11 \\
\hline
\end{tabular}

Notes. ${ }^{(a)}$ The value of $r_{\text {in }}$ for this observation corresponds to a $5 \%$ pile-up fraction.

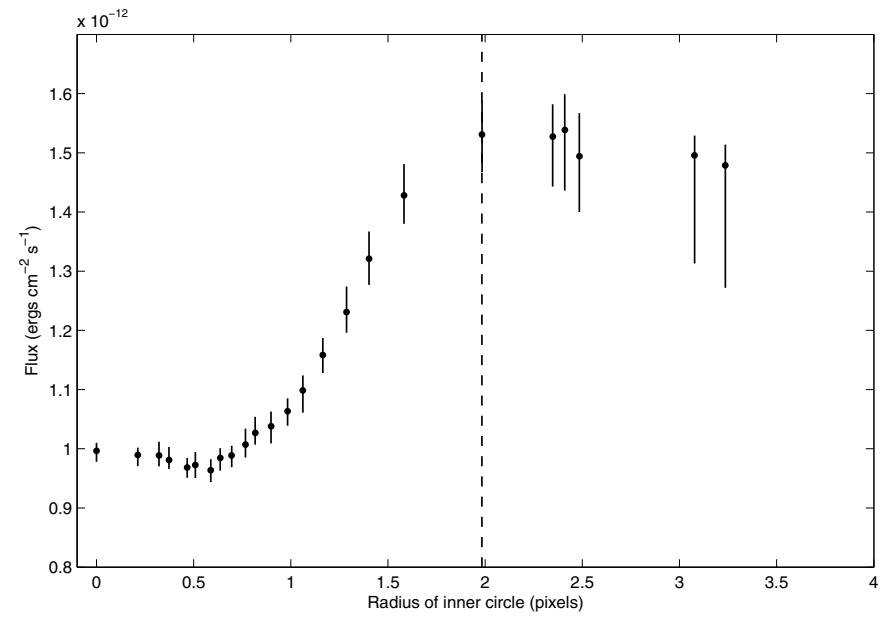

Fig. A.2. Inferred flux of NGC 4278 during the observation 7078 as a function of $r_{\text {in }}$ following the technique for a series of excluded core circles. The vertical dashed line corresponds to the $\sim 2 \%$ pile-up fraction. The flux increases to reach a plateau when the piled up core is discarded.

\section{Appendix B: Surrounding medium around NGC 4278 nucleus}

We turn now to study the environment around the nucleus of NGC 4278. Two sources, labeled source 1 and source 2 in Fig. 2 (hereinafter, S1 and S2), within a $10^{\prime \prime}$-radius circle around the nucleus were detected in the six observations and therefore complete X-ray spectral study can be possible. We consider the rest of the medium as diffuse emission plus emission from point-like sources detected in five of the six observations or less. Source events and spectra were extracted, for both S1 and S2, from a polygonal shape around the sources centroids that encircles $90 \%$ of the PSF. Background annular regions were constructed in the same manner as for the nucleus (see Sect. 2.1). The spectrum and the background for the diffuse emission is constructed as explained in the AE users guide Sect. 7.1.2.

We fit the six spectra for each of the two sources, S1 and S2, simultaneously with a power-law model (Figs. B.1 and B.2 respectively). Both fit are acceptable with reduced $\chi^{2}$ of 1.06 and 0.8 for 43 and 27 d.o.f. for S1 and S2 respectively. The fit results in a power-law photon index of $1.0 \pm 0.2$ and $1.3_{-0.4}^{+0.7}$ for $\mathrm{S} 1$ and $\mathrm{S} 2$ respectively. The $0.5-8 \mathrm{keV}$ absorption corrected fluxes

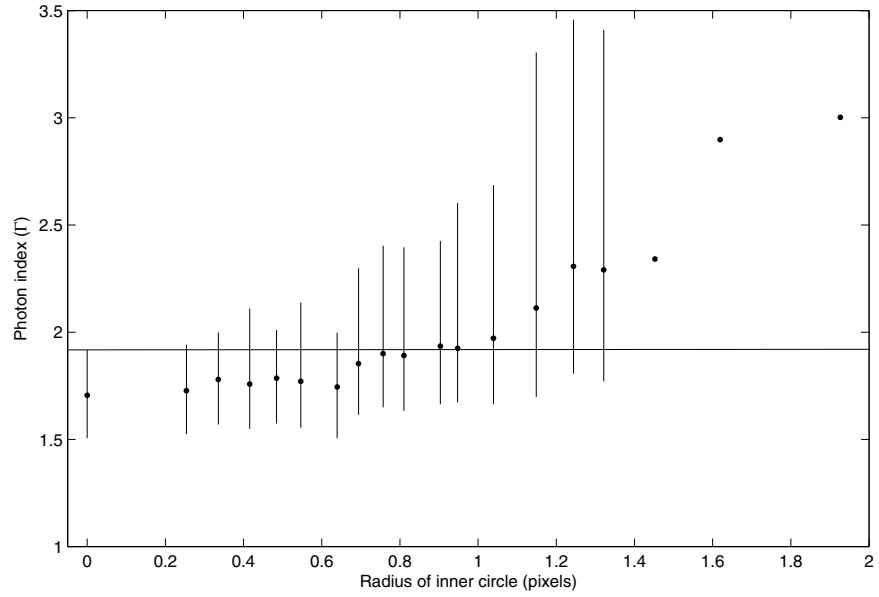

Fig. A.3. The photon index, $\Gamma$, as a function of $r_{\text {in }}$ (obs. ID: 398). Although it is clear that there is a tendency for $\Gamma$ to increase going away from the PSF center, the different values are within the error bars.

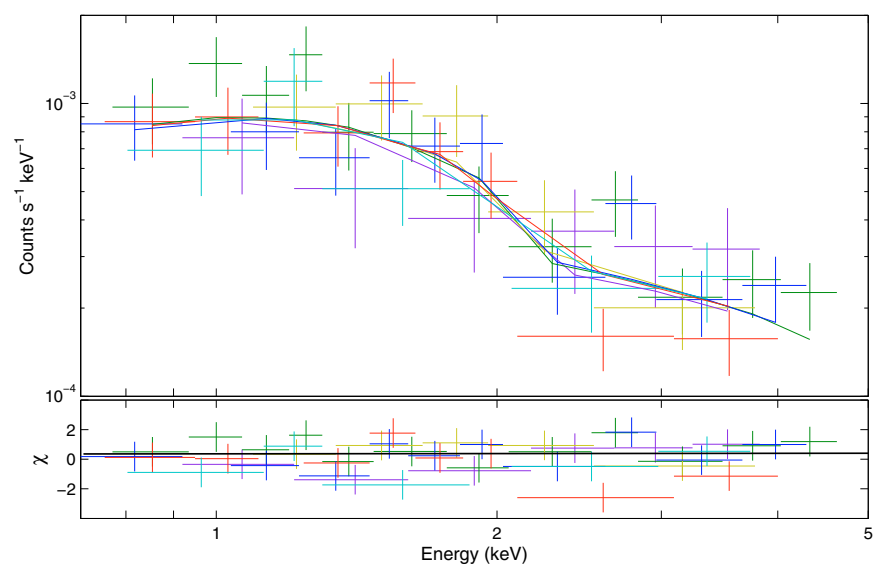

Fig. B.1. The best fit absorbed power-law model to S1 spectra.

are $2.6_{-0.3}^{+0.2} \times 10^{-14} \mathrm{erg} \mathrm{cm}^{-2} \mathrm{~s}^{-1}$ and $0.6_{-0.2}^{+0.1} \times 10^{-14} \mathrm{erg} \mathrm{cm}^{-2} \mathrm{~s}^{-1}$ for $S 1$ and $S 2$ respectively. Adopting the same distance to the nucleus of 16.7 Mpc (Tonry et al. 2001), this results in a corrected luminosities of $8.7 \times 10^{38}$ and $2 \times 10^{38} \mathrm{erg} \mathrm{s}^{-1}$ for S1 and S2 respectively. The power-law spectral fit and the luminosities derived for these two sources make them compatible with the 


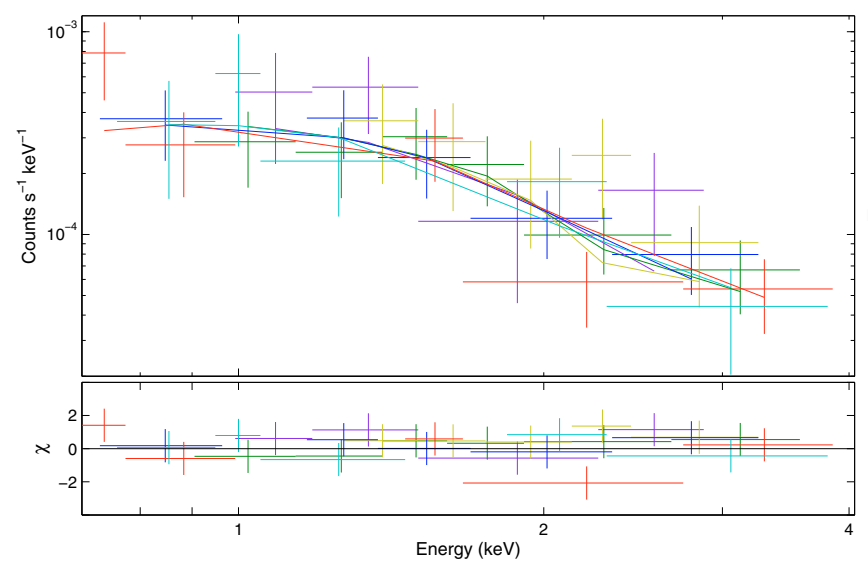

Fig. B.2. The best fit absorbed power-law model to S2 spectra.

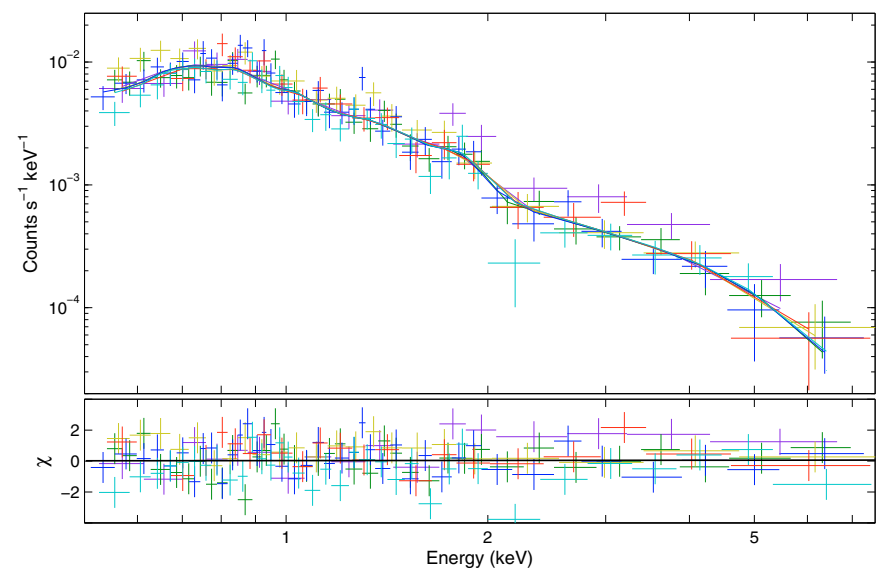

Fig. B.3. The best fit absorbed power-law plus a thermal component to the diffuse emission spectra.

low mass X-ray binary populations in elliptical galaxies (see Fabbiano 2006, for a review). The hydrogen column density of the intrinsic absorption affecting the power-law model has an upper limit of $\sim 10^{21} \mathrm{~cm}^{-2}$ for both S1 and S2. We note that a disk black-body model can fit the spectra as well with an inner disk temperature of 1.2 and $2.9 \mathrm{keV}$ for $\mathrm{S} 1$ and $\mathrm{S} 2$ respectively.

The diffuse emission is fit with a combination of an absorbed power-law and a thermal mekal component (Fig. B.3). We find a reduced $\chi^{2}$ of 1.3 for 136 d.o.f. The power-law photon index, $\Gamma$, is $1.8 \pm 0.2$ whereas the temperature of the thermal component is $0.4 \pm 0.1 \mathrm{keV}$. The discrepancy of this thermal temperature to the nucleus thermal temperature obtained with Chandra inside a radius of $\sim 3^{\prime \prime}$ could be the fact that the spectra derived for the diffuse emission includes part of the less-hot interstellar medium at a larger distance from the nucleus $\left(10^{\prime \prime}\right)$. The intrinsic absorption derived from the diffuse emission fit has an upper limit of $\sim 10^{21} \mathrm{~cm}^{-2}$. The corrected $0.5-8 \mathrm{keV}$ flux is $5.3 \times 10^{-14} \mathrm{erg} \mathrm{cm}^{-2} \mathrm{~s}^{-1}$ which gives a corrected luminosity of $17.7 \times 10^{38} \mathrm{erg} \mathrm{s}^{-1}$.

The total $0.5-8 \mathrm{keV}$ corrected luminosity of the two pointlike sources and the diffuse emission is $28.4 \times 10^{38} \mathrm{erg} \mathrm{s}^{-1}$. This corresponds to $4 \%$ of the nuclear flux during the brightest Chandra observation (obs. ID 4741) and to $12 \%$ during the faintest one (obs. ID 7081). Now if we assume that the raise in the flux during the XMM-Newton observation is due to a burst in one of the X-ray point-like sources, $\mathrm{S} 1$ or $\mathrm{S} 2$, then the flux of these objects should increase, in about 8 months period, by a factor of 50 or 200, respectively.

To check if such an increase in one of the sources is qualitatively possible we calculated the Eddington luminosity of S1 and S2 assuming that these sources are neutron star LMXBs with a neutron star mass of about $1.4 M_{\odot}$. This gives a $L_{\text {edd }}$ of about $1.75 \times 10^{38} \mathrm{erg} \mathrm{s}^{-1}$ which leads to a super Eddington ratio, $L_{\mathrm{X}} / L_{\text {edd }}$, of 250 . Therefore, we can completely discard the idea of an increase in the X-ray flux of S1 and S2 that resulted in the high flux observed during the XMM-Newton observation. Hence, The raise of the flux in the case of the $X M M-N e w t o n$ observation is definitely due to variation from the LLAGN X-ray emission. Consequently, S1, S2, and the diffuse emission contribute to only $2 \%$ of the total EPIC flux that would explain the less-needed thermal component in the XMM-Newton $\mathrm{X}$-ray spectral fit as the emission is almost totally dominated by the LLAGN.

\section{References}

Alonso-Herrero, A., Rieke, M. J., Rieke, G. H., \& Shields, J. C. 2000, ApJ, 530, 688

Arnaud, K. A. 1996, in Astronomical Data Analysis Software and Systems V, ASP Conf. Ser., 101, 17

Brassington, N. J., Fabbiano, G., Kim, D.-W., et al. 2009, ApJS, 181, 605 Brenneman, L. W., Weaver, K. A., Kadler, M., et al. 2009, ApJ, 698, 528

Broos, P. S., Townsley, L. K., \& Nousek, J. A. 1998, in SPIE Conf. Ser. 3444, ed. R. B. Hoover, \& A. B. Walker, 30

Broos, P. S., Townsley, L. K., Feigelson, E. D., et al. 2010, ApJ, 714, 1582

Capetti, A., de Ruiter, H. R., Fanti, R., et al. 2000, A\&A, 362, 871 Capetti, A., Celotti, A., Chiaberge, M., et al. 2002, A\&A, 383, 104 Cardelli, J. A., Clayton, G. C., \& Mathis, J. S. 1989, ApJ, 345, 245 Cardullo, A., Corsini, E. M., Beifiori, A., et al. 2009, A\&A, 508, 641 Chiaberge, M., Capetti, A., \& Macchetto, F. D. 2005, ApJ, 625, 716 Davis, J. E. 2001, ApJ, 562, 575

de Vaucouleurs, G., de Vaucouleurs, A., Corwin, Jr., H. G., et al. 1991, Third Reference Catalogue of Bright Galaxies, ed. G. de Vaucouleurs, et al.

Elvis, M., Wilkes, B. J., McDowell, J. C., et al. 1994, ApJS, 95, 1

Eracleous, M., Hwang, J. A., \& Flohic, H. M. L. G. 2010, ApJS, 187, 135 Fabbiano, G. 2006, ARA\&A, 44, 323

Flohic, H. M. L. G., Eracleous, M., Chartas, G., Shields, J. C., \& Moran, E. C. 2006, ApJ, 647, 140

Freeman, P. E., Kashyap, V., Rosner, R., \& Lamb, D. Q. 2002, ApJS, 138, 185

Gammie, C. F., Narayan, R., \& Blandford, R. 1999, ApJ, 516, 177

Getman, K. V., Flaccomio, E., Broos, P. S., et al. 2005, ApJS, 160, 319

Giroletti, M., Taylor, G. B., \& Giovannini, G. 2005, ApJ, 622, 178

González-Martín, O., Masegosa, J., Márquez, I., Guainazzi, M., \& Jiménez-Bailón, E. 2009, A\&A, 506, 1107

Guainazzi, M., Matt, G., \& Perola, G. C. 2005, A\&A, 444, 119

Heckman, T. M. 1980, A\&A, 87, 152

Heckman, T. M., Lebofsky, M. J., Rieke, G. H., \& van Breugel, W. 1983, ApJ, 272, 400

Ho, L. C. 1999, ApJ, 516, 672

Ho, L. C. 2002, in Issues in Unification of Active Galactic Nuclei, ed. R. Maiolino, A. Marconi, \& N. Nagar, ASP Conf. Ser., 258, 165

Ho, L. C. 2005, Ap\&SS, 300, 219

Ho, L. C. 2008, ARA\&A, 46, 475

Ho, L. C., Filippenko, A. V., \& Sargent, W. L. W. 1993, ApJ, 417, 63

Ho, L. C., Filippenko, A. V., Sargent, W. L. W., \& Peng, C. Y. 1997, ApJS, 112, 391

Ho, L. C., Feigelson, E. D., Townsley, L. K., et al. 2001, ApJ, 549, L51

Jiménez-Bailón, E., Piconcelli, E., Guainazzi, M., et al. 2005, A\&A, 435, 449

Jones, D. L., Wrobel, J. M., \& Shaffer, D. B. 1984, ApJ, 276, 480

Kalberla, P. M. W., Burton, W. B., Hartmann, D., et al. 2005, A\&A, 440, 775

Kim, D., \& Fabbiano, G. 2004, ApJ, 611, 846

Koratkar, A., \& Blaes, O. 1999, PASP, 111, 1

Lauer, T. R., Faber, S. M., Gebhardt, K., et al. 2005, AJ, 129, 2138

Longmore, A. J., \& Sharples, R. M. 1982, MNRAS, 201, 111

Lu, Y., \& Yu, Q. 2001, MNRAS, 324, 653 
Malkan, M. A., \& Sargent, W. L. W. 1982, ApJ, 254, 22

Manmoto, T., Takeuchi, M., Mineshige, S., Matsumoto, R., \& Negoro, H. 1996, ApJ, 464, L135

Maoz, D. 2007, MNRAS, 377, 1696

Maoz, D., Nagar, N. M., Falcke, H., \& Wilson, A. S. 2005, ApJ, 625, 699

Mason, K. O., Breeveld, A., Much, R., et al. 2001, A\&A, 365, L36

Mewe, R., Gronenschild, E. H. B. M., \& van den Oord, G. H. J. 1985, A\&AS, 62, 197

Nagar, N. M., Wilson, A. S., \& Falcke, H. 2001, ApJ, 559, L87

Nagar, N. M., Falcke, H., Wilson, A. S., \& Ulvestad, J. S. 2002, A\&A, 392, 53

Nagar, N. M., Falcke, H., \& Wilson, A. S. 2005, A\&A, 435, 521

Nandra, K., George, I. M., Mushotzky, R. F., Turner, T. J., \& Yaqoob, T. 1997, ApJ, 476, 70

Nandra, K., O’Neill, P. M., George, I. M., \& Reeves, J. N. 2007, MNRAS, 382, 194

Narayan, R. 2005, Ap\&SS, 300, 177

Negoro, H., Miyamoto, S., \& Kitamoto, S. 1994, ApJ, 423, L127

Nemmen, R. S., Storchi-Bergmann, T., Yuan, F., et al. 2006, ApJ, 643, 652

Nemmen, R. S., Storchi-Bergmann, T., Eracleous, M., \& Yuan, F. 2010, IAU Symp., 267, 313

Papadakis, I. E., Ioannou, Z., Brinkmann, W., \& Xilouris, E. M. 2008, A\&A, 490, 995

Pian, E., Romano, P., Maoz, D., et al. 2010, MNRAS, 401, 677
Porquet, D., Reeves, J. N., O’Brien, P., \& Brinkmann, W. 2004, A\&A, 422, 85 Ptak, A., Yaqoob, T., Mushotzky, R., Serlemitsos, P., \& Griffiths, R. 1998, ApJ, 501, L37

Ptak, A., Terashima, Y., Ho, L. C., \& Quataert, E. 2004, ApJ, 606, 173

Quataert, E., Di Matteo, T., Narayan, R., \& Ho, L. C. 1999, ApJ, 525, L89

Reeves, J. N., Wynn, G., O’Brien, P. T., \& Pounds, K. A. 2002, MNRAS, 336, L56

Sanders, D. B., Phinney, E. S., Neugebauer, G., Soifer, B. T., \& Matthews, K. 1989, ApJ, 347, 29

Sikora, M., Stawarz, Ł., \& Lasota, J. 2007, ApJ, 658, 815

Steffen, A. T., Strateva, I., Brandt, W. N., et al. 2006, AJ, 131, 2826

Strüder, L., Briel, U., Dennerl, K., et al. 2001, A\&A, 365, L18

Takeuchi, M., \& Mineshige, S. 1997, ApJ, 486, 160

Terashima, Y., \& Wilson, A. S. 2003, ApJ, 583, 145

Terashima, Y., Ho, L. C., \& Ptak, A. F. 2000, ApJ, 539, 161

Terashima, Y., Iyomoto, N., Ho, L. C., \& Ptak, A. F. 2002, ApJS, 139, 1

Tonry, J. L., Dressler, A., Blakeslee, J. P., et al. 2001, ApJ, 546, 681

Tremaine, S., Gebhardt, K., Bender, R., et al. 2002, ApJ, 574, 740

Turner, T. J., George, I. M., Nandra, K., \& Turcan, D. 1999, ApJ, 524, 667

Turner, M. J. L., Abbey, A., Arnaud, M., et al. 2001, A\&A, 365, L27

Wang, T., \& Zhang, X. 2003, MNRAS, 340, 793

Weisskopf, M. C., Brinkman, B., Canizares, C., et al. 2002, PASP, 114, 1

Wilms, J., Allen, A., \& McCray, R. 2000, ApJ, 542, 914 